MATHEMATICS OF COMPUTATION

Volume 74, Number 250, Pages 629-653

S 0025-5718(04)01677-1

Article electronically published on May 25, 2004

\title{
LINEAR DIFFERENCE EQUATIONS WITH TRANSITION POINTS
}

\author{
Z. WANG AND R. WONG
}

\begin{abstract}
Two linearly independent asymptotic solutions are constructed for the second-order linear difference equation

$$
y_{n+1}(x)-\left(A_{n} x+B_{n}\right) y_{n}(x)+y_{n-1}(x)=0,
$$

where $A_{n}$ and $B_{n}$ have power series expansions of the form

$$
A_{n} \sim \sum_{s=0}^{\infty} \frac{\alpha_{s}}{n^{s}}, \quad B_{n} \sim \sum_{s=0}^{\infty} \frac{\beta_{s}}{n^{s}}
$$

with $\alpha_{0} \neq 0$. Our results hold uniformly for $x$ in an infinite interval containing the transition point $x_{+}$given by $\alpha_{0} x_{+}+\beta_{0}=2$. As an illustration, we present an asymptotic expansion for the monic polynomials $\pi_{n}(x)$ which are orthogonal with respect to the modified Jacobi weight $w(x)=(1-x)^{\alpha}(1+x)^{\beta} h(x)$, $x \in(-1,1)$, where $\alpha, \beta>-1$ and $h$ is real analytic and strictly positive on $[-1,1]$.
\end{abstract}

\section{INTRODUCTION}

Ever since Deift and Zhou 7] introduced the steepest descent method for Riemann-Hilbert problems, there has been a considerable amount of activities in the study of asymptotics of orthogonal polynomials by using this approach. For instance, in [5] Deift et al. studied the asymptotics of orthogonal polynomials with respect to the weight $w(x)=e^{-Q(x)}$ on the real line, where $Q(x)$ is a polynomial of even degree with positive leading coefficient, and they obtained uniform PlancherelRotach-type asymptotics in the entire complex plane. Also, in 11 Kuijlaars and McLaughlin used this method to investigate the asymptotic behavior of Laguerre polynomials $L_{n}^{\left(\alpha_{n}\right)}(x)$, where $\alpha_{n}$ is a sequence of negative numbers such that $-\alpha_{n} / n$ tends to a limit $A>1$ as $n \rightarrow \infty$. Furthermore, Kuijlaars et al. 12, 13 considered the asymptotics of the polynomials that are orthogonal with respect to the modified Jacobi weight

$$
w(x)=(1-x)^{\alpha}(1+x)^{\beta} h(x), \quad x \in(-1,1),
$$

where $\alpha, \beta>-1$ and the extra factor $h$ is real analytic and strictly positive on $[-1,1]$. For other investigations of a similar nature, we refer to [3, 4, 6, 10, 18, An advantage of this new approach over the more classical asymptotic methods is that it

Received by the editor April 2, 2003 and, in revised form, October 6, 2003.

2000 Mathematics Subject Classification. Primary 41A60, 39A10, 33C45.

Key words and phrases. Difference equation, transition points, three-term recurrence relation, orthogonal polynomials.

The work of this author was partially supported by the Research Grant Council of Hong Kong under Project 9040522 . 
is applicable to orthogonal polynomials which do not have an integral representation or satisfy a second-order differential equation. Examples of such cases are provided by the two sets of orthogonal polynomials mentioned above, namely those associated with (i) the exponential weight and (ii) the modified Jacobi weight. However, so far this new method has not been able to produce results as strong as those obtainable from the classical approaches when an integral representation is available or when the differential equation theory can be applied. For instance, in the case of MeixnerPollaczek polynomials $M_{n}(x ; \delta, \eta)$, one can use a Cauchy integral representation to derive an infinite asymptotic expansion for $M_{n}(\alpha n ; \delta, \eta)$, which holds uniformly for $-M \leq \alpha \leq M$, where $M$ can be any positive number; see 14. Also, when the polynomial $Q(x)=x^{2 m}+\cdots$ in the weight function $w(x)=e^{-Q(x)}$ is even and convex, one can use the turning point theory for differential equations to obtain an asymptotic formula for the polynomials $p_{n}(x)$ orthogonal with respect to $w(x)$, which holds uniformly in the unbounded interval $0 \leq x \leq O\left(n^{1 / 2 m}\right)$; see [16].

In our view, a desirable approach to derive asymptotic expansions for orthogonal polynomials now is to develop an asymptotic theory for linear second-order difference equations, just like what Langer, Cherry, Olver and others have done for linear differential equations; see the definitive book by Olver [15]. Our view is based on the fact that any sequence of orthogonal polynomials satisfies the three-term recurrence relation

$$
p_{n+1}(x)=\left(a_{n} x+b_{n}\right) p_{n}(x)-c_{n} p_{n-1}(x), \quad n=1,2, \ldots,
$$

where $a_{n}, b_{n}$ and $c_{n}$ are constants. If we define a sequence $\left\{K_{n}\right\}$ recursively by $K_{n+1} / K_{n-1}=c_{n}$, with $K_{0}$ and $K_{1}$ depending on the particular sequence of polynomials, and if we put $A_{n} \equiv a_{n} K_{n} / K_{n+1}, B_{n} \equiv b_{n} K_{n} / K_{n+1}$ and $P_{n}(x) \equiv p_{n}(x) / K_{n}$, then (1.2) can be written as

$$
P_{n+1}(x)-\left(A_{n} x+B_{n}\right) P_{n}(x)+P_{n-1}(x)=0 .
$$

We shall assume that the coefficients $A_{n}$ and $B_{n}$ are real and have asymptotic expansions of the form

$$
A_{n} \sim n^{-\theta} \sum_{s=0}^{\infty} \frac{\alpha_{s}}{n^{s}} \quad \text { and } \quad B_{n} \sim \sum_{s=0}^{\infty} \frac{\beta_{s}}{n^{s}},
$$

where $\theta$ is a real number and $\alpha_{0} \neq 0$. If $x$ is a fixed number, then asymptotic solutions to (1.3) can be obtained from existing results in the literature; see, e.g., the papers by Birkhoff [1] and Birkhoff and Trjitzinsky [2. These papers, however, have been considered far too complicated and even impenetrable. For a more accessible account of the asymptotic behavior of the solutions to equation (1.3), we refer to the two more recent papers by Wong and $\mathrm{Li}$ [21] 22]. When $x$ is a parameter and is allowed to vary, then not much work has been done in this area until just recently. In [20, we have studied a case in which the exponent $\theta$ in (1.4) is not zero. This case corresponds to the turning-point problem for secondorder linear differential equations, and the asymptotic expansions derived for the solutions involve the Airy functions $\operatorname{Ai}(\cdot), \operatorname{Bi}(\cdot)$ and their derivatives; see also [19]. In this paper, we shall consider the case $\theta=0$ in (1.3). The approximants of the asymptotic solutions turn out to be Bessel functions or modified Bessel functions. As an illustration, we shall present an infinite asymptotic expansion for the monic polynomials which are orthogonal with respect to the modified Jacobi weight given in (1.1). Our expansion will hold uniformly for $x$ in $[-1+\delta, \infty), \delta>0$. Although 
we will make use of a result of Kuijlaars et al. [12] on the coefficients of a relevant recurrence relation, our result for the modified Jacobi polynomials is stronger than those given in their papers [12, 13].

The presentation of this paper is arranged as follows. In Section 2, we show how the Bessel functions arise in the asymptotic solutions. In Section 3, we give a preliminary lemma which is crucial to the derivation of the asymptotic expansions. The construction of the formal solutions is presented in Section 4. In Section 5, we establish the asymptotic nature of the expansions. The final section is devoted to a study of the orthogonal polynomials with the modified Jacobi weight.

\section{Motivation LeAding to the EXPANSiOn}

Returning to (1.3), we try a solution of the form $P_{n}(x)=\lambda^{n}$ and replace the coefficients $A_{n}$ and $B_{n}$ by their respective asymptotic expansions given in (1.4) with $\theta=0$. Upon letting $n \rightarrow \infty$, this yields the characteristic equation

$$
\lambda^{2}-\left(\alpha_{0} x+\beta_{0}\right) \lambda+1=0 .
$$

The roots of this equation are

$$
\lambda_{ \pm}=\frac{1}{2}\left[\alpha_{0} x+\beta_{0} \pm \sqrt{\left(\alpha_{0} x+\beta_{0}\right)^{2}-4}\right],
$$

and they coincide when $x=x_{ \pm}$, where $x_{ \pm}$satisfy

$$
\alpha_{0} x_{ \pm}+\beta_{0}= \pm 2 .
$$

The points $x_{+}$and $x_{-}$are called transition points in [20]. Throughout this paper, we shall assume that

$$
\alpha_{1}=\beta_{1}=0 .
$$

This assumption naturally implies the condition

$$
\alpha_{1} x_{+}+\beta_{1}=0,
$$

which was used in our previous paper on turning point theory; see equation (2.7) in [20. It is interesting to note that in a paper on WKB methods for difference equations, Dingle and Morgan 8, 9] also assumed this condition. In fact, they assumed the stronger condition that all coefficients $\alpha_{s}$ and $\beta_{s}$ in (1.4) with odd indices vanish. (We believe that under assumption (1.4), condition (2.4) for the three-term recurrence relation (1.3) is probably satisfied by all orthogonal polynomials in the Szegö class. This possibility is currently under investigation.) Note that since $\widetilde{P}_{n}(x) \equiv(-1)^{n} P_{n}(x)$ satisfies the recurrence relation $\widetilde{P}_{n+1}(x)+\left(A_{n} x+B_{n}\right) \widetilde{P}_{n}(x)+\widetilde{P}_{n-1}(x)=0$, we may, without loss of generality, assume $\alpha_{0}>0$.

Now let $\tau_{0}:=-\left(\alpha_{3} x_{+}+\beta_{3}\right) / 2\left(\alpha_{2} x_{+}+\beta_{2}\right)$; cf. (4.20) below. Define $N:=n+\tau_{0}$ and recast the expansions in (1.4), with $\theta=0$, in terms of $N$. This gives

$$
A_{n} \sim \sum_{s=0}^{\infty} \frac{\alpha_{s}^{\prime}}{N^{s}} \quad \text { and } \quad B_{n} \sim \sum_{s=0}^{\infty} \frac{\beta_{s}^{\prime}}{N^{s}}
$$

with $\alpha_{0}^{\prime}=\alpha_{0}>0$ and $\alpha_{1}^{\prime}=\beta_{1}^{\prime}=0$. Since the transition points $x_{+}$and $x_{-}$in (2.3) are distinct, we may restrict ourselves to just the case $x=x_{+}$. As in [20], we try a 
formal solution of the form

$$
P_{n}(x)=\sum_{s=0}^{\infty} X_{s}(\xi) N^{-s}
$$

for $x$ near $x_{+}$, where $\xi$ depends on $x$ and $N$. In this paper, we choose $\xi=N \zeta^{1 / 2}(x)$, where $\zeta(x)$ is an increasing function with $\zeta\left(x_{+}\right)=0$. Clearly,

$$
\begin{aligned}
P_{n+1}(x) & =\sum_{s=0}^{\infty} X_{s}\left[(N+1) \zeta^{1 / 2}(x)\right](N+1)^{-s} \\
& =\sum_{s=0}^{\infty} X_{s}\left[\left(1+\frac{1}{N}\right) \xi\right] N^{-s}\left(1+\frac{1}{N}\right)^{-s} .
\end{aligned}
$$

By expanding $X_{s}\left(\xi+\frac{\xi}{N}\right)$ into a Taylor series and using the binomial expansion, we obtain

$$
P_{n+1}(x)=\sum_{l=0}^{\infty} \frac{1}{N^{l}} \sum_{s=0}^{l}\left[\sum_{j=0}^{l-s} X_{s}^{(j)}(\xi) \frac{\xi^{j}}{j !}\left(\begin{array}{c}
-s \\
l-j-s
\end{array}\right)\right] .
$$

Similarly,

$$
P_{n-1}(x)=\sum_{l=0}^{\infty} \frac{1}{N^{l}} \sum_{s=0}^{l}\left[(-1)^{l-s} \sum_{j=0}^{l-s} X_{s}^{(j)}(\xi) \frac{\xi^{j}}{j !}\left(\begin{array}{c}
-s \\
l-j-s
\end{array}\right)\right] .
$$

Since $\xi=N \zeta^{1 / 2}(x)$, we also have

$$
x=\zeta^{-1}\left(\xi^{2} / N^{2}\right)=\sum_{j=0}^{\infty} \frac{1}{j !}\left(D^{j} \zeta^{-1}\right)(0) \frac{\xi^{2 j}}{N^{2 j}},
$$

where $D^{j}$ denotes the $j$-th derivative of $\zeta^{-1}$. From (2.6) and (2.10), it follows that

$$
A_{n} x+B_{n}=\sum_{s=0}^{\infty} \frac{Q_{s}(\xi)}{N^{s}},
$$

where $Q_{0}(\xi)=\alpha_{0}^{\prime} x_{+}+\beta_{0}^{\prime}=2, Q_{1}(\xi)=\alpha_{1}^{\prime} x_{+}+\beta_{1}^{\prime}=0$ by $(2.5)$, and

$$
Q_{s}(\xi)=\beta_{s}^{\prime}+\sum_{2 j \leq s} \frac{1}{j !}\left(D^{j} \zeta^{-1}\right)(0) \alpha_{s-2 j}^{\prime} \xi^{2 j}, \quad s=2,3, \ldots
$$

Upon substituting $(2.7),(2.8),(2.9)$ and (2.12) into (1.3), we find that $X_{0}(\xi)$ satisfies the Bessel equation

$$
\frac{d^{2} X_{0}}{d \xi^{2}}=\left(\frac{\alpha_{0}^{\prime}}{\zeta^{\prime}(0)}+\frac{\alpha_{2}^{\prime} x_{+}+\beta_{2}^{\prime}}{\xi^{2}}\right) X_{0} .
$$

Thus, it follows that $X_{0}(\xi)$ can be expressed in terms of either the Bessel functions $J_{\nu}(\xi)$ and $Y_{\nu}(\xi)$ or the modified Bessel functions $I_{\nu}(\xi)$ and $K_{\nu}(\xi)$. That is, there are constants $C_{1}$ and $C_{2}$ such that

$$
X_{0}(\xi)=C_{1} \xi^{1 / 2} J_{\nu}(\xi)+C_{2} \xi^{1 / 2} Y_{\nu}(\xi) \quad \text { if } \quad \alpha_{0}^{\prime}<0,
$$

and

$$
X_{0}(\xi)=C_{1} \xi^{1 / 2} I_{\nu}(\xi)+C_{2} \xi^{1 / 2} K_{\nu}(\xi) \quad \text { if } \quad \alpha_{0}^{\prime}>0
$$


where

$$
\nu= \pm\left(\alpha_{2}^{\prime} x_{+}+\beta_{2}^{\prime}+\frac{1}{4}\right)^{1 / 2} .
$$

Under the assumption in (2.4), it is easily verified that $\alpha_{2}^{\prime}=\alpha_{2}$ and $\beta_{2}^{\prime}=\beta_{2}$. In view of the well-known identities

$$
\begin{array}{cc}
J_{-\nu}(z)=\cos \nu \pi J_{\nu}(z)-\sin \nu \pi Y_{\nu}(z), & I_{-\nu}(z)=I_{\nu}(z)+\frac{2 \sin \nu \pi}{\pi} K_{\nu}(z), \\
Y_{-\nu}(z)=\sin \nu \pi J_{\nu}(z)+\cos \nu \pi Y_{\nu}(z) & \text { and } \quad K_{-\nu}(z)=K_{\nu}(z),
\end{array}
$$

we may, without loss of generality, take the square root with the + sign in $(2.14)$. Moreover, each of the subsequent coefficient functions $X_{s}(\xi), s=1,2, \ldots$, in $(2.7)$ satisfies an inhomogeneous Bessel equation. This suggests that instead of $(2.7)$, we might as well try the formal series solution

$$
P_{n}(x)=Z_{\nu}\left(N \zeta^{1 / 2}\right) \sum_{s=0}^{\infty} \frac{\bar{A}_{s}(\zeta)}{N^{s-\frac{1}{2}}}+Z_{\nu-1}\left(N \zeta^{1 / 2}\right) \sum_{s=0}^{\infty} \frac{\bar{B}_{s}(\zeta)}{N^{s-\frac{1}{2}}}
$$

motivated from the differential equation theory. In $(2.15), Z_{\nu}(\xi)$ can be any solution of the modified Bessel equation

$$
y^{\prime \prime}+\frac{1}{x} y^{\prime}-\left(1+\frac{\nu^{2}}{x^{2}}\right) y=0 .
$$

The main result of this paper is given in the following theorem.

Theorem. Assume that the coefficients $A_{n}$ and $B_{n}$ in the recurrence relation (1.3) are real, and have asymptotic expansions given in (1.4) with $\theta=0$. Let $x_{ \pm}$be the transition points defined in (2.3),

$$
\zeta^{1 / 2}=\cosh ^{-1}\left(\alpha_{0} x+\beta_{0}\right) / 2 \quad \text { and } \quad \tau_{0}=-\left(\alpha_{3} x_{+}+\beta_{3}\right) / 2\left(\alpha_{2} x_{+}+\beta_{2}\right) .
$$

Then, for each nonnegative integer p, equation (1.3) has a pair of linearly independent solutions

$$
\begin{aligned}
P_{n}(x)=\left(\frac{4 \zeta}{\left(\alpha_{0} x+\beta_{0}\right)^{2}-4}\right)^{\frac{1}{4}} & {\left[N^{\frac{1}{2}} I_{\nu}\left(N \zeta^{\frac{1}{2}}\right) \sum_{s=0}^{p} \frac{A_{s}(\zeta)}{N^{s}}\right.} \\
& \left.+N^{\frac{1}{2}} \zeta^{\frac{1}{2}} I_{\nu-1}\left(N \zeta^{\frac{1}{2}}\right) \sum_{s=0}^{p} \frac{B_{s}(\zeta)}{N^{s}}+\varepsilon_{p}(N, x)\right]
\end{aligned}
$$

and

$$
\begin{aligned}
Q_{n}(x)=\left(\frac{4 \zeta}{\left(\alpha_{0} x+\beta_{0}\right)^{2}-4}\right)^{\frac{1}{4}} & {\left[N^{\frac{1}{2}} K_{\nu}\left(N \zeta^{\frac{1}{2}}\right) \sum_{s=0}^{p} \frac{A_{s}(\zeta)}{N^{s}}\right.} \\
& \left.-N^{\frac{1}{2}} \zeta^{\frac{1}{2}} K_{\nu-1}\left(N \zeta^{\frac{1}{2}}\right) \sum_{s=0}^{p} \frac{B_{s}(\zeta)}{N^{s}}+\delta_{p}(N, x)\right],
\end{aligned}
$$

where $N=n+\tau_{0}$ and $\nu$ is given in (2.14). The error terms satisfy

$$
\left|\varepsilon_{p}(N, x)\right| \leq \frac{M_{p}}{N^{p+\frac{1}{2}}}\left[\left|I_{\nu}\left(N \zeta^{\frac{1}{2}}\right)\right|+\left|I_{\nu-1}\left(N \zeta^{\frac{1}{2}}\right)\right|\right]
$$


and

$$
\left|\delta_{p}(N, x)\right| \leq \frac{M_{p}}{N^{p+\frac{1}{2}}}\left[\left|K_{\nu}\left(N \zeta^{\frac{1}{2}}\right)\right|+\left|K_{\nu-1}\left(N \zeta^{\frac{1}{2}}\right)\right|\right]
$$

for $x_{-}+\delta \leq x<\infty$, where $M_{p}$ is a positive constant. The coefficients $A_{s}(\zeta)$ and $B_{s}(\zeta)$ can be determined successively for any given $A_{0}(\zeta)$ and $B_{0}(\zeta)$; see $(4.11)$ and (4.12).

To see how the function $\zeta(x)$ and the constant $\tau_{0}$ in the above theorem are chosen, we refer to Section 4; see, in particular, (4.8) and (4.20).

\section{A PRELIMINARY LEMma}

When we replace $n$ by $n+1$, the two functions $Z_{\nu}\left(N \zeta^{1 / 2}\right)$ and $Z_{\nu-1}\left(N \zeta^{1 / 2}\right)$ in (2.15) become $Z_{\nu}\left[(N+1) \zeta^{1 / 2}\right]$ and $Z_{\nu-1}\left[(N+1) \zeta^{1 / 2}\right]$. An important connection between the second two functions and the first two is given in the following lemma, which plays a crucial role in the derivation of the formal series solution (2.15).

Lemma 1. Let $Z_{\nu}(x)$ be any solution of the modified Bessel equation (2.16), which satisfies

$$
Z_{\nu-1}(x)=Z_{\nu}^{\prime}(x)+\left(\frac{\nu}{x}\right) Z_{\nu}(x)
$$

We have

$\left(1+\frac{\theta}{N}\right)^{1 / 2} Z_{\nu}\left[(N+\theta) \zeta^{1 / 2}\right]=Z_{\nu}\left(N \zeta^{1 / 2}\right) G(N ; \theta, \zeta)+\zeta^{1 / 2} Z_{\nu-1}\left(N \zeta^{1 / 2}\right) H(N ; \theta, \zeta)$

and

$\left(1+\frac{\theta}{N}\right)^{1 / 2} Z_{\nu-1}\left[(N+\theta) \zeta^{1 / 2}\right]=\zeta^{1 / 2} Z_{\nu}\left(N \zeta^{1 / 2}\right) L(N ; \theta, \zeta)+Z_{\nu-1}\left(N \zeta^{1 / 2}\right) K(N ; \theta, \zeta)$,

where

$$
\begin{aligned}
G(N ; \theta, \zeta) & \sim \sum_{s=0}^{\infty} \frac{G_{s}(\theta, \zeta)}{N^{s}}, & H(N ; \theta, \zeta) & \sim \sum_{s=0}^{\infty} \frac{H_{s}(\theta, \zeta)}{N^{s}}, \\
L(N ; \theta, \zeta) & \sim \sum_{s=0}^{\infty} \frac{L_{s}(\theta, \zeta)}{N^{s}}, & K(N ; \theta, \zeta) & \sim \sum_{s=0}^{\infty} \frac{K_{s}(\theta, \zeta)}{N^{s}},
\end{aligned}
$$

the expansions being uniformly valid with respect to bounded $\theta$ and all real $\zeta$.

Proof. Let $w(\theta, \zeta)=(1+\theta / N)^{1 / 2} Z_{\nu}\left[(N+\theta) \zeta^{1 / 2}\right]$. Straightforward calculation gives

$$
\frac{\partial w}{\partial \theta}=\frac{\frac{1}{2}-\nu}{N}\left(1+\frac{\theta}{N}\right)^{-\frac{1}{2}} Z_{\nu}+\left(1+\frac{\theta}{N}\right)^{\frac{1}{2}} \zeta^{\frac{1}{2}} Z_{\nu-1}
$$

and

$$
\frac{\partial^{2} w}{\partial \theta^{2}}=\left(\zeta+\frac{\nu^{2}-\frac{1}{4}}{(N+\theta)^{2}}\right) w
$$


The last equation can also be obtained directly from (2.16) by eliminating the term involving the first derivative. From Taylor's expansion,

$$
w(\theta, \zeta)=w(0, \zeta)+\frac{\partial w}{\partial \theta}(0, \zeta) \theta+\frac{1}{2 !} \frac{\partial^{2} w}{\partial \theta^{2}}(0, \zeta) \theta^{2}+\cdots .
$$

In view of (3.6) and (3.7), this series can be rearranged as

$$
\left(1+\frac{\theta}{N}\right)^{\frac{1}{2}} Z_{\nu}\left[(N+\theta) \zeta^{\frac{1}{2}}\right]=Z_{\nu}\left(N \zeta^{\frac{1}{2}}\right) G(N ; \theta, \zeta)+\zeta^{\frac{1}{2}} Z_{\nu-1}\left(N \zeta^{\frac{1}{2}}\right) H(N ; \theta, \zeta),
$$

where $G(N ; 0, \zeta)=1, H(N ; 0, \zeta)=0$,

$$
\frac{\partial G}{\partial \theta}(N ; 0, \zeta)=\frac{\frac{1}{2}-\nu}{N} \quad \text { and } \quad \frac{\partial H}{\partial \theta}(N ; 0, \zeta)=1 .
$$

Differentiating (3.8) with respect to $\theta$ yields

$$
\frac{\partial^{2} G}{\partial \theta^{2}}=\left(\zeta+\frac{\nu^{2}-\frac{1}{4}}{(N+\theta)^{2}}\right) G,\left.\quad G\right|_{\theta=0}=1,\left.\quad \frac{\partial G}{\partial \theta}\right|_{\theta=0}=\frac{\frac{1}{2}-\nu}{N}
$$

and

$$
\frac{\partial^{2} H}{\partial \theta^{2}}=\left(\zeta+\frac{\nu^{2}-\frac{1}{4}}{(N+\theta)^{2}}\right) H,\left.\quad H\right|_{\theta=0}=0,\left.\quad \frac{\partial H}{\partial \theta}\right|_{\theta=0}=1 .
$$

The solutions to these two differential equations have formal asymptotic solutions

$$
G=\sum_{s=0}^{\infty} \frac{G_{s}(\theta, \zeta)}{N^{s}} \quad \text { and } \quad H=\sum_{s=0}^{\infty} \frac{H_{s}(\theta, \zeta)}{N^{s}}
$$

where the coefficients can be determined recursively by the equations

$$
\begin{aligned}
& \left\{\begin{array}{lll}
\frac{\partial^{2} G_{0}}{\partial \theta^{2}}-\zeta G_{0}=0, & \left.G_{0}\right|_{\theta=0}=1, & \left.\frac{\partial G_{0}}{\partial \theta}\right|_{\theta=0}=0, \\
\frac{\partial^{2} H_{0}}{\partial \theta^{2}}-\zeta H_{0}=0, & \left.H_{0}\right|_{\theta=0}=0, & \left.\frac{\partial H_{0}}{\partial \theta}\right|_{\theta=0}=1,
\end{array}\right. \\
& \left\{\begin{array}{lll}
\frac{\partial^{2} G_{1}}{\partial \theta^{2}}-\zeta G_{1}=0, & \left.G_{1}\right|_{\theta=0}=0, & \left.\frac{\partial G_{1}}{\partial \theta}\right|_{\theta=0}=\frac{1}{2}-\nu, \\
\frac{\partial^{2} H_{1}}{\partial \theta^{2}}-\zeta H_{1}=0, & \left.H_{1}\right|_{\theta=0}=0, & \left.\frac{\partial H_{1}}{\partial \theta}\right|_{\theta=0}=0,
\end{array}\right. \\
& \left\{\begin{array}{l}
\frac{\partial^{2} G_{s}}{\partial \theta^{2}}-\zeta G_{s}=\left(\nu^{2}-\frac{1}{4}\right) \sum_{j=2}^{s}(-1)^{j}(j-1) \theta^{j-2} G_{s-j} \\
\left.G_{s}\right|_{\theta=0}=\left.\frac{\partial G_{s}}{\partial \theta}\right|_{\theta=0}=0
\end{array}\right.
\end{aligned}
$$

and

$$
\left\{\begin{array}{l}
\frac{\partial^{2} H_{s}}{\partial \theta^{2}}-\zeta H_{s}=\left(\nu^{2}-\frac{1}{4}\right) \sum_{j=2}^{s}(-1)^{j}(j-1) \theta^{j-2} H_{s-j} \\
\left.H_{s}\right|_{\theta=0}=\left.\frac{\partial H_{s}}{\partial \theta}\right|_{\theta=0}=0
\end{array}\right.
$$


for $s \geq 2$. These equations can be solved explicitly, and we have

$$
\begin{aligned}
G_{0} & =\frac{1}{2}\left(e^{\sqrt{\zeta} \theta}+e^{-\sqrt{\zeta} \theta}\right), & H_{0} & =\frac{1}{2 \sqrt{\zeta}}\left(e^{\sqrt{\zeta} \theta}-e^{-\sqrt{\zeta} \theta}\right), \\
G_{1} & =\frac{\frac{1}{2}-\nu}{2 \sqrt{\zeta}}\left(e^{\sqrt{\zeta} \theta}-e^{-\sqrt{\zeta} \theta}\right), & H_{1} & =0,
\end{aligned}
$$

and

$$
\left\{\begin{aligned}
G_{s} & =\frac{\nu^{2}-\frac{1}{4}}{2 \sqrt{\zeta}} \int_{0}^{\theta}\left(\sum_{j=2}^{s}(-1)^{j}(j-1) \phi^{j-2} G_{s-j}\right)\left(e^{\sqrt{\zeta}(\theta-\phi)}-e^{\sqrt{\zeta}(\phi-\theta)}\right) d \phi \\
H_{s} & =\frac{\nu^{2}-\frac{1}{4}}{2 \sqrt{\zeta}} \int_{0}^{\theta}\left(\sum_{j=2}^{s}(-1)^{j}(j-1) \phi^{j-2} H_{s-j}\right)\left(e^{\sqrt{\zeta}(\theta-\phi)}-e^{\sqrt{\zeta}(\phi-\theta)}\right) d \phi
\end{aligned}\right.
$$

for $s \geq 2$, where $\sqrt{\zeta}=i \sqrt{-\zeta}$ if $\zeta<0$. Using the inequalities

$$
\cosh x \sinh y \leq y \cosh (x+y) \quad \text { and } \quad \sinh x \sinh y \leq y \sinh (x+y),
$$

it can be proved by induction that for $\zeta<0$

$$
\left|G_{s}(\theta, \zeta)\right| \leq(|\nu|+1)^{s}|\theta|^{s} \quad \text { and } \quad\left|H_{s}(\theta, \zeta)\right| \leq(|\nu|+1)^{s}|\theta|^{s+1},
$$

and that for $\zeta>0$

$$
\left|G_{s}(\theta, \zeta)\right| \leq(|\nu|+1)^{s}|\theta|^{s} G_{0}(\theta, \zeta), \quad\left|H_{s}(\theta, \zeta)\right| \leq(|\nu|+1)^{s}|\theta|^{s}\left|H_{0}(\theta, \zeta)\right| .
$$

Thus, the formal series in (3.11) are uniformly convergent for any bounded $\theta$ and sufficiently large $N$, and (3.2) follows.

Let $L_{s}(\theta, \zeta)$ and $K_{s}(\theta, \zeta)$ be given as in (3.5). By the same argument, we have

$$
\begin{gathered}
K_{0}(\theta, \zeta)=\frac{1}{2}\left(e^{\sqrt{\zeta} \theta}+e^{-\sqrt{\zeta} \theta}\right), \quad L_{0}(\theta, \zeta)=\frac{1}{2 \sqrt{\zeta}}\left(e^{\sqrt{\zeta} \theta}-e^{-\sqrt{\zeta} \theta}\right), \\
K_{1}(\theta, \zeta)=\frac{\nu-\frac{1}{2}}{2 \sqrt{\zeta}}\left(e^{\sqrt{\zeta} \theta}-e^{-\sqrt{\zeta} \theta}\right), \quad L_{1}(\theta, \zeta)=0 .
\end{gathered}
$$

Furthermore, for $\zeta<0$

$$
\left|K_{s}(\theta, \zeta)\right| \leq(|\nu|+1)^{s}|\theta|^{s}, \quad\left|L_{s}(\theta, \zeta)\right| \leq(|\nu|+1)^{s}|\theta|^{s+1},
$$

and for $\zeta>0$

$$
\left|K_{s}(\theta, \zeta)\right| \leq(|\nu|+1)^{s}|\theta|^{s} K_{0}(\theta, \zeta), \quad\left|L_{s}(\theta, \zeta)\right| \leq(|\nu|+1)^{s}|\theta|^{s}\left|L_{0}(\theta, \zeta)\right| .
$$

This demonstrates the uniform convergence of the formal series in (3.5) for sufficiently large $N$ and completes the proof of the lemma.

From the recursive formula (3.16)-(3.18), it can be readily shown that

$$
G_{s}(-\theta, \zeta)=(-1)^{s} G_{s}(\theta, \zeta) \text { and } H_{s}(-\theta, \zeta)=(-1)^{s-1} H_{s}(\theta, \zeta) .
$$

Choosing $\theta= \pm 1$ in (3.2), we have

$$
\left(1 \pm \frac{1}{N}\right)^{\frac{1}{2}} Z_{\nu}\left[(N \pm 1) \zeta^{\frac{1}{2}}\right]=Z_{\nu}\left(N \zeta^{\frac{1}{2}}\right) G\left(\zeta, \pm \frac{1}{N}\right) \pm \zeta^{\frac{1}{2}} Z_{\nu-1}\left(N \zeta^{\frac{1}{2}}\right) H\left(\zeta, \pm \frac{1}{N}\right)
$$

where

$$
G\left(\zeta, \pm \frac{1}{N}\right):=G(N ; \pm 1, \zeta):=\sum_{s=0}^{\infty}( \pm 1)^{s} \frac{G_{s}(\zeta)}{N^{s}}
$$


and

$$
H\left(\zeta, \pm \frac{1}{N}\right):= \pm H(N ; \pm 1, \zeta):=\sum_{s=0}^{\infty}( \pm 1)^{s} \frac{H_{s}(\zeta)}{N^{s}}
$$

Similarly, it follows from (3.3) that

$$
\left(1 \pm \frac{1}{N}\right)^{\frac{1}{2}} Z_{\nu-1}\left[(N \pm 1) \zeta^{\frac{1}{2}}\right]= \pm \zeta^{\frac{1}{2}} Z_{\nu}\left(N \zeta^{\frac{1}{2}}\right) L\left(\zeta, \pm \frac{1}{N}\right)+Z_{\nu-1}\left(N \zeta^{\frac{1}{2}}\right) K\left(\zeta, \pm \frac{1}{N}\right)
$$

where

$$
L\left(\zeta, \pm \frac{1}{N}\right):= \pm L(N ; \pm 1, \zeta):=\sum_{s=0}^{\infty}( \pm 1)^{s} \frac{L_{s}(\zeta)}{N^{s}}
$$

and

$$
K\left(\zeta, \pm \frac{1}{N}\right):=K(N ; \pm 1, \zeta):=\sum_{s=0}^{\infty}( \pm 1)^{s} \frac{K_{s}(\zeta)}{N^{s}}
$$

By (3.16) and (3.21), we also have

$$
G_{0}(\zeta)=K_{0}(\zeta)=\cosh \zeta^{\frac{1}{2}}
$$

and

$$
H_{0}(\zeta)=L_{0}(\zeta)=\frac{\sinh \sqrt{\zeta}}{\sqrt{\zeta}}
$$

Later in our discussion, we also need the values $G\left(0, \frac{1}{N}\right), H\left(0, \frac{1}{N}\right), L\left(0, \frac{1}{N}\right)$ and $K\left(0, \frac{1}{N}\right)$. To this end, we note that using (3.26) with $Z_{\nu}=I_{\nu}$ and $Z_{\nu}=e^{i \nu \pi} K_{\nu}$, we have, respectively,

$$
G\left(\zeta, \frac{1}{N}\right)=\left(1+\frac{1}{N}\right)^{\frac{1}{2}} \frac{K_{\nu}\left[(N+1) \zeta^{\frac{1}{2}}\right]}{K_{\nu}\left(N \zeta^{\frac{1}{2}}\right)}+\frac{\zeta^{\frac{1}{2}} K_{\nu-1}\left(N \zeta^{\frac{1}{2}}\right)}{K_{\nu}\left(N \zeta^{\frac{1}{2}}\right)} H\left(\zeta, \frac{1}{N}\right)
$$

and

$$
H\left(\zeta, \frac{1}{N}\right)=\left(1+\frac{1}{N}\right)^{\frac{1}{2}} \frac{I_{\nu}\left[(N+1) \zeta^{\frac{1}{2}}\right]}{\zeta^{\frac{1}{2}} I_{\nu-1}\left(N \zeta^{\frac{1}{2}}\right)}-\frac{I_{\nu}\left(N \zeta^{\frac{1}{2}}\right)}{\zeta^{\frac{1}{2}} I_{\nu-1}\left(N \zeta^{\frac{1}{2}}\right)} G\left(\zeta, \frac{1}{N}\right)
$$

From the asymptotic relations

$$
I_{\nu}(z) \sim \frac{(z / 2)^{\nu}}{\Gamma(\nu+1)} \text { and } K_{\nu}(z) \sim \frac{1}{2} \Gamma(\nu)\left(\frac{z}{2}\right)^{-\nu}
$$

as $z \rightarrow 0$, it follows that

$$
\begin{gathered}
G\left(0, \frac{1}{N}\right)=\left(1+\frac{1}{N}\right)^{-\nu+\frac{1}{2}}, \\
H\left(0, \frac{1}{N}\right)=\frac{N}{2 \nu}\left[\left(1+\frac{1}{N}\right)^{\nu+\frac{1}{2}}-\left(1+\frac{1}{N}\right)^{-\nu+\frac{1}{2}}\right] .
\end{gathered}
$$


In a similar manner, we obtain

$$
\begin{gathered}
K\left(0, \frac{1}{N}\right)=\left(1+\frac{1}{N}\right)^{\nu-\frac{1}{2}} \\
L\left(0, \frac{1}{N}\right)=\frac{N}{2(\nu-1)}\left[\left(1+\frac{1}{N}\right)^{\nu-\frac{1}{2}}-\left(1+\frac{1}{N}\right)^{-\nu+\frac{3}{2}}\right] .
\end{gathered}
$$

\section{Formal ASYmptotic solutions}

Let $\zeta(x)$ be an increasing function with $\zeta\left(x_{+}\right)=0$. We try a formal series solution to (1.3) in the form

$$
P_{n}(x)=N^{\frac{1}{2}} Z_{\nu}\left(N \zeta^{\frac{1}{2}}\right) \sum_{s=0}^{\infty} \frac{A_{s}(\zeta)}{N^{s}}+N^{\frac{1}{2}} \zeta^{\frac{1}{2}} Z_{\nu-1}\left(N \zeta^{\frac{1}{2}}\right) \sum_{s=0}^{\infty} \frac{B_{s}(\zeta)}{N^{s}} ;
$$

cf. (2.15). For convenience, we put

$$
A\left(\zeta, \frac{1}{N}\right):=\sum_{s=0}^{\infty} \frac{A_{s}(\zeta)}{N^{s}}, \quad B\left(\zeta, \frac{1}{N}\right):=\sum_{s=0}^{\infty} \frac{B_{s}(\zeta)}{N^{s}}
$$

and

$$
\Psi\left(x, \frac{1}{N}\right):=A_{n} x+B_{n}=\sum_{s=0}^{\infty} \frac{\alpha_{s}^{\prime} x+\beta_{s}^{\prime}}{N^{s}} ;
$$

cf. (1.3) and (2.6). By Lemma 1, we have

$$
\begin{aligned}
& P_{n \pm 1}(x)=N^{\frac{1}{2}} Z_{\nu}\left(N \zeta^{\frac{1}{2}}\right)\left\{G\left(\zeta, \pm \frac{1}{N}\right) A\left(\zeta, \frac{1}{N \pm 1}\right) \pm \zeta L\left(\zeta, \pm \frac{1}{N}\right) B\left(\zeta, \frac{1}{N \pm 1}\right)\right\} \\
& +N^{\frac{1}{2}} \zeta^{\frac{1}{2}} Z_{\nu-1}\left(N \zeta^{\frac{1}{2}}\right)\left\{K\left(\zeta, \pm \frac{1}{N}\right) B\left(\zeta, \frac{1}{N \pm 1}\right) \pm H\left(\zeta, \pm \frac{1}{N}\right) A\left(\zeta, \frac{1}{N \pm 1}\right)\right\} .
\end{aligned}
$$

Substituting (4.1) and (4.4) into the recurrence relation (1.3) and matching the coefficients of $Z_{\nu}$ and $Z_{\nu-1}$, we obtain

$$
\begin{array}{r}
G\left(\zeta, \frac{1}{N}\right) A\left(\zeta, \frac{1}{N+1}\right)+G\left(\zeta,-\frac{1}{N}\right) A\left(\zeta, \frac{1}{N-1}\right)-\Psi\left(x, \frac{1}{N}\right) A\left(\zeta, \frac{1}{N}\right) \\
+\zeta L\left(\zeta, \frac{1}{N}\right) B\left(\zeta, \frac{1}{N+1}\right)-\zeta L\left(\zeta,-\frac{1}{N}\right) B\left(\zeta, \frac{1}{N-1}\right)=0
\end{array}
$$

and

$$
\begin{aligned}
H\left(\zeta, \frac{1}{N}\right) A\left(\zeta, \frac{1}{N+1}\right) & -H\left(\zeta,-\frac{1}{N}\right) A\left(\zeta, \frac{1}{N-1}\right)-\Psi\left(x, \frac{1}{N}\right) B\left(\zeta, \frac{1}{N}\right) \\
& +K\left(\zeta, \frac{1}{N}\right) B\left(\zeta, \frac{1}{N+1}\right)+K\left(\zeta,-\frac{1}{N}\right) B\left(\zeta, \frac{1}{N-1}\right)=0 .
\end{aligned}
$$

By letting $N \rightarrow \infty$, the last two equations give

$$
G_{0}(\zeta)=K_{0}(\zeta)=\frac{\alpha_{0}^{\prime} x+\beta_{0}^{\prime}}{2} .
$$


Coupling (3.16) and (4.7) yields

$$
\zeta^{\frac{1}{2}}=\cosh ^{-1}\left(\frac{\alpha_{0}^{\prime} x+\beta_{0}^{\prime}}{2}\right) .
$$

Equating coefficients of like powers of $1 / N$ in (4.5) and (4.6), we get

$$
\begin{aligned}
\sum_{2 s \leq p} & {\left[A_{p-2 s} \sum_{i=0}^{2 s}\left(\begin{array}{c}
2 s-p \\
2 s-i
\end{array}\right) G_{i}\right] } \\
& +\sum_{2 s+1 \leq p}\left[B_{p-2 s-1} \sum_{i=0}^{2 s+1}\left(\begin{array}{c}
2 s+1-p \\
2 s+1-i
\end{array}\right) \zeta L_{i}\right]-\sum_{s \leq p} \frac{\alpha_{s}^{\prime} x+\beta_{s}^{\prime}}{2} A_{p-s}=0
\end{aligned}
$$

and

$$
\begin{aligned}
\sum_{2 s \leq p} & {\left[B_{p-2 s} \sum_{i=0}^{2 s}\left(\begin{array}{c}
2 s-p \\
2 s-i
\end{array}\right) K_{i}\right] } \\
& +\sum_{2 s+1 \leq p}\left[A_{p-2 s-1} \sum_{i=0}^{2 s+1}\left(\begin{array}{c}
2 s+1-p \\
2 s+1-i
\end{array}\right) H_{i}\right]-\sum_{s \leq p} \frac{\alpha_{s}^{\prime} x+\beta_{s}^{\prime}}{2} B_{p-s}=0 .
\end{aligned}
$$

From (4.9) and (4.10), it follows that

$$
\begin{aligned}
(p-1) H_{0} A_{p-1}= & \sum_{1 \leq 2 s \leq p}\left[B_{p-2 s} \sum_{i=0}^{2 s}\left(\begin{array}{c}
2 s-p \\
2 s-i
\end{array}\right) K_{i}\right] \\
& +\sum_{2 \leq 2 s+1 \leq p}\left[A_{p-2 s-1} \sum_{i=0}^{2 s+1}\left(\begin{array}{c}
2 s+1-p \\
2 s+1-i
\end{array}\right) H_{i}\right] \\
& -\sum_{2 \leq s \leq p} \frac{\alpha_{s}^{\prime} x+\beta_{s}^{\prime}}{2} B_{p-s}
\end{aligned}
$$

and

$$
\begin{aligned}
(p-1) \zeta L_{0} B_{p-1}= & \sum_{1 \leq 2 s \leq p}\left[A_{p-2 s} \sum_{i=0}^{2 s}\left(\begin{array}{c}
2 s-p \\
2 s-i
\end{array}\right) G_{i}\right] \\
& +\sum_{2 \leq 2 s+1 \leq p}\left[B_{p-2 s-1} \sum_{i=0}^{2 s+1}\left(\begin{array}{c}
2 s+1-p \\
2 s+1-i
\end{array}\right) \zeta L_{i}\right] \\
& -\sum_{2 \leq s \leq p} \frac{\alpha_{s}^{\prime} x+\beta_{s}^{\prime}}{2} A_{p-s},
\end{aligned}
$$

where we have made use of the fact that $H_{1}=L_{1}=0$. Thus, for each $p \geq 1, A_{p}(\zeta)$ and $B_{p}(\zeta)$ can be determined successively from the above two equations for any given $A_{0}(\zeta)$ and $B_{0}(\zeta)$.

Lemma 2. Let $\zeta(x)$ be as in (4.8), and suppose $\left|A_{0}(\zeta)\right|$ and $\left(1+|\zeta|^{1 / 2}\right)\left|B_{0}(\zeta)\right|$ are bounded for $x \geq x_{-}+\delta$. Furthermore, let $A_{s}(\zeta)$ and $B_{s}(\zeta)$ be successively defined as in (4.11) and (4.12). Then there exists a positive constant $N_{s}$ independent of $x$ such that

$$
\left|A_{s}(\zeta)\right| \leq N_{s}, \quad\left|B_{s}(\zeta)\right| \leq \frac{N_{s}}{\left(1+|\zeta|^{1 / 2}\right)}, \quad s=1,2, \ldots,
$$

for all $x \geq x_{-}+\delta, \delta>0$. 
Proof. Using (3.32) and (3.33), it can be shown that

$$
\left|G_{0}\right| \leq C\left|H_{0}\right|\left(1+|\zeta|^{1 / 2}\right)
$$

for $x \geq x_{-}+\delta$, where $C$ is a positive constant. From (3.19), (3.20), (3.23) and (3.24), it also follows that there exists a constant $C_{s}$, independent of $x$, such that

$$
\left|K_{s}\right|+\left|G_{s}\right| \leq C_{s}\left|H_{0}\right|\left(1+|\zeta|^{1 / 2}\right) .
$$

Thus, if the functions $A_{p}(\zeta)$ and $B_{p}(\zeta)$ given successively in (4.11) and (4.12) are well-defined (i.e., $B_{p}(\zeta)$ is bounded at $\zeta=0$ ), then the estimates in (4.13) can be readily verified by induction. To show that $B_{p}(0)$ is bounded, we divide our discussion into three cases: (i) $2 \nu \neq 0,1,2, \ldots$, (ii) $2 \nu=1,2,3, \ldots$, and (iii) $2 \nu=0$.

In case (i), we first consider the second-order linear difference equation

$$
y_{N+1}+y_{N-1}-\Psi\left(x_{+}, \frac{1}{N}\right) y_{N}=0,
$$

where $\Psi\left(x_{+}, 1 / N\right)$ is given in (4.3). The results in 21] infer that (4.16) has two linearly independent asymptotic solutions of the form

$$
y_{N}^{(1)} \sim N^{-\nu+\frac{1}{2}} \sum_{s=0}^{\infty} \frac{c_{s}}{N^{s}}, \quad y_{N}^{(2)} \sim N^{\nu+\frac{1}{2}} \sum_{s=0}^{\infty} \frac{d_{s}}{N^{s}},
$$

where $c_{0}=d_{0}=1$,

$$
c_{1}=\frac{\alpha_{3}^{\prime} x_{+}+\beta_{3}^{\prime}}{1+2 \nu} \quad \text { and } \quad d_{1}=\frac{\alpha_{3}^{\prime} x_{+}+\beta_{3}^{\prime}}{1-2 \nu}
$$

on account of (2.14). We shall show that for all $p \geq 0$,

$$
A_{p}(0)=c_{p} \quad \text { and } \quad B_{p}(0)=\frac{d_{p+1}-c_{p+1}}{2 \nu} .
$$

To this end, note that

$$
\alpha_{3}^{\prime} x_{+}+\beta_{3}^{\prime}=\alpha_{3} x_{+}+\beta_{3}+2 \tau_{0}\left(\alpha_{2} x_{+}+\beta_{2}\right) .
$$

Since $2 \nu \neq 0,1,2, \ldots$ in this case, we can choose $\tau_{0}$ to be

$$
\tau_{0}=-\frac{\alpha_{3} x_{+}+\beta_{3}}{2\left(\alpha_{2} x_{+}+\beta_{2}\right)}=-\frac{\alpha_{3} x_{+}+\beta_{3}}{2\left(\nu^{2}-\frac{1}{4}\right)},
$$

so that $B_{0}(0)=0$. Note that $\nu \neq 1 / 2$ in the present case and that the last equality follows from (2.14).

Returning to (4.5) and (4.6), we set $\zeta=0$. This yields

$$
G\left(0, \frac{1}{N}\right) A\left(0, \frac{1}{N+1}\right)+G\left(0,-\frac{1}{N}\right) A\left(0, \frac{1}{N-1}\right)-\Psi\left(x_{+}, \frac{1}{N}\right) A\left(0, \frac{1}{N}\right)=0
$$

and

$$
\begin{array}{r}
H\left(0, \frac{1}{N}\right) A\left(0, \frac{1}{N+1}\right)-H\left(0,-\frac{1}{N}\right) A\left(0, \frac{1}{N-1}\right)-\Psi\left(x_{+}, \frac{1}{N}\right) B\left(0, \frac{1}{N}\right) \\
+K\left(0, \frac{1}{N}\right) B\left(0, \frac{1}{N+1}\right)+K\left(0,-\frac{1}{N}\right) B\left(0, \frac{1}{N-1}\right)=0 .
\end{array}
$$


To see that (4.21) can be written in the form of (4.16), we put

$$
x_{N}:=N^{-\nu+\frac{1}{2}} A\left(0, \frac{1}{N}\right) \text {. }
$$

From (4.21) and (3.34), it is clear that $x_{N}$ satisfies

$$
x_{N+1}+x_{N-1}-\Psi\left(x_{+}, \frac{1}{N}\right) x_{N}=0
$$

and we have

$$
N^{-\nu+\frac{1}{2}} A\left(0, \frac{1}{N}\right)=y_{N}^{(1)} \sim N^{-\nu+\frac{1}{2}} \sum_{s=0}^{\infty} \frac{c_{s}}{N^{s}}
$$

on account of (4.17). This gives the first equation in (4.19). Analogously, we put

$$
\hat{x}_{N}=N^{\nu-\frac{1}{2}}\left[N A\left(0, \frac{1}{N}\right)+2 \nu B\left(0, \frac{1}{N}\right)\right] \text {. }
$$

Then, by using (3.35) and (3.36), it can be shown that $\hat{x}_{N}$ satisfies (4.16) and

$$
N^{\nu-\frac{1}{2}}\left[N A\left(0, \frac{1}{N}\right)+2 \nu B\left(0, \frac{1}{N}\right)\right]=y_{N}^{(2)} \sim N^{\nu+\frac{1}{2}} \sum_{s=0}^{\infty} \frac{d_{s}}{N^{s}},
$$

from which

$$
A_{s}(0)+2 \nu B_{s-1}(0)=d_{s}
$$

follows and we obtain the second equation in (4.19).

In case (ii), i.e., $2 \nu=1,2, \ldots$, the two linearly independent asymptotic solutions of (4.16) are

$$
y_{N}^{(1)} \sim N^{-\nu+\frac{1}{2}} \sum_{s=0}^{\infty} \frac{c_{s}}{N^{s}}, \quad y_{N}^{(2)} \sim N^{\nu+\frac{1}{2}} \sum_{s=0}^{\infty} \frac{d_{s}}{N^{s}}+C y_{N}^{(1)} \log N,
$$

where $C$ is a constant; see Wong and Li 21 . In a similar manner, we have

$$
N^{-\nu+\frac{1}{2}} A\left(0, \frac{1}{N}\right)=y_{N}^{(1)} \sim N^{-\nu+\frac{1}{2}} \sum_{s=0}^{\infty} \frac{c_{s}}{N^{s}}
$$

and

$$
N^{\nu-\frac{1}{2}}\left[N A\left(0, \frac{1}{N}\right)+2 \nu B\left(0, \frac{1}{N}\right)\right]=0
$$

on account of (4.26). Coupling these together gives

$$
A_{s}(0)=c_{s} \quad \text { for } s=0,1, \ldots
$$

and

$$
2 \nu B_{s}(0)=A_{s+1}(0) \quad \text { for } s=0,1, \ldots
$$

When $\nu \neq 1 / 2$, we can still choose $\tau_{0}$ as in (4.20) so that $B_{0}(0)=0$. When $\nu=1 / 2$, there is no singularity at $\xi=0$ in (2.13), and the functions $Z_{\nu}$ and $Z_{\nu-1}$ in (4.1) can be expressed in terms of hyperbolic cosine and hyperbolic sine. So $\nu=1 / 2$ is a simple case, and we can choose $\tau_{0}$ to be any real number.

In case (iii), $\nu=0$ and the results in 21 infer that (4.16) has two linearly independent asymptotic solutions of the form

$$
y_{N}^{(1)} \sim N^{\frac{1}{2}} \sum_{s=0}^{\infty} \frac{c_{s}}{N^{s}}, \quad y_{N}^{(2)} \sim N^{\frac{1}{2}} \sum_{s=0}^{\infty} \frac{d_{s}}{N^{s}}+y_{N}^{(1)} \log N,
$$


where $c_{0}=d_{0}=1$ and $c_{1}=-d_{1}=\left(\alpha_{3}^{\prime} x_{+}+\beta_{3}^{\prime}\right)$. Similarly, we have

$$
N^{\frac{1}{2}} A\left(0, \frac{1}{N}\right)=y_{N}^{(1)} \sim N^{\frac{1}{2}} \sum_{s=0}^{\infty} \frac{c_{s}}{N^{s}}
$$

and

$$
N^{-\frac{1}{2}}\left[(N \log N) A\left(0, \frac{1}{N}\right)+B\left(0, \frac{1}{N}\right)\right]=y_{N}^{(2)}-y_{N}^{(1)} \sim N^{\frac{1}{2}} \sum_{s=1}^{\infty} \frac{d_{s}-c_{s}}{N^{s}}+y_{N}^{(1)} \log N,
$$

from which it follows

$$
A_{s}(0)=c_{s} \quad \text { and } \quad B_{s}(0)=d_{s+1}-c_{s+1} \quad \text { for } \quad s=0,1, \ldots
$$

In the case of $\nu=0$, we can also choose $\tau_{0}$ as in (4.20) so that $B_{0}(0)=0$. The proof of the lemma is now complete.

\section{Proof OF THE THEOREM}

Since $x$ is a fixed number in the recurrence relation (1.3), we may take

$$
Z_{\nu}\left(N \zeta^{\frac{1}{2}}\right)=H_{0}^{-\frac{1}{2}}(\zeta) I_{\nu}\left(N \zeta^{\frac{1}{2}}\right) \quad \text { and } \quad Z_{\nu}\left(N \zeta^{\frac{1}{2}}\right)=e^{i \pi \nu} H_{0}^{-\frac{1}{2}}(\zeta) K_{\nu}\left(N \zeta^{\frac{1}{2}}\right)
$$

in (4.1). In view of (3.33) and (4.8), we obtain two formal solutions

$$
\begin{aligned}
P_{n}(x)=\left(\frac{4 \zeta}{\left(\alpha_{0}^{\prime} x+\beta_{0}^{\prime}\right)^{2}-4}\right)^{\frac{1}{4}}[ & N^{\frac{1}{2}} I_{\nu}\left(N \zeta^{\frac{1}{2}}\right) \sum_{s=0}^{\infty} \frac{A_{s}(\zeta)}{N^{s}} \\
& \left.+N^{\frac{1}{2}} \zeta^{\frac{1}{2}} I_{\nu-1}\left(N \zeta^{\frac{1}{2}}\right) \sum_{s=0}^{\infty} \frac{B_{s}(\zeta)}{N^{s}}\right]
\end{aligned}
$$

and

$$
\begin{aligned}
Q_{n}(x)=\left(\frac{4 \zeta}{\left(\alpha_{0}^{\prime} x+\beta_{0}^{\prime}\right)^{2}-4}\right)^{\frac{1}{4}}[ & N^{\frac{1}{2}} K_{\nu}\left(N \zeta^{\frac{1}{2}}\right) \sum_{s=0}^{\infty} \frac{A_{s}(\zeta)}{N^{s}} \\
& \left.-N^{\frac{1}{2}} \zeta^{\frac{1}{2}} K_{\nu-1}\left(N \zeta^{\frac{1}{2}}\right) \sum_{s=0}^{\infty} \frac{B_{s}(\zeta)}{N^{s}}\right] .
\end{aligned}
$$

Here, $I_{\nu}$ and $K_{\nu}$ are the modified Bessel functions.

For convenience, we introduce the notations

$$
\begin{array}{r}
A_{p}\left(\zeta, \frac{1}{N}\right):=\sum_{s=0}^{p} \frac{A_{s}(\zeta)}{N^{s}}, \quad B_{p}\left(\zeta, \frac{1}{N}\right):=\sum_{s=0}^{p} \frac{B_{s}(\zeta)}{N^{s}} \\
r_{n}^{p}(x):=\left(\frac{4 \zeta}{\left(\alpha_{0}^{\prime} x+\beta_{0}^{\prime}\right)^{2}-4}\right)^{\frac{1}{4}}\left[N^{\frac{1}{2}} I_{\nu}\left(N \zeta^{\frac{1}{2}}\right) A_{p}\left(\zeta, \frac{1}{N}\right)\right. \\
\left.+N^{\frac{1}{2}} \zeta^{\frac{1}{2}} I_{\nu-1}\left(N \zeta^{\frac{1}{2}}\right) B_{p}\left(\zeta, \frac{1}{N}\right)\right]
\end{array}
$$

and

$$
\begin{aligned}
s_{n}^{p}(x):=\left(\frac{4 \zeta}{\left(\alpha_{0}^{\prime} x+\beta_{0}^{\prime}\right)^{2}-4}\right)^{\frac{1}{4}} & {\left[N^{\frac{1}{2}} K_{\nu}\left(N \zeta^{\frac{1}{2}}\right) A_{p}\left(\zeta, \frac{1}{N}\right)\right.} \\
& \left.-N^{\frac{1}{2}} \zeta^{\frac{1}{2}} K_{\nu-1}\left(N \zeta^{\frac{1}{2}}\right) B_{p}\left(\zeta, \frac{1}{N}\right)\right] .
\end{aligned}
$$


By Lemma 1, we have

$$
r_{n+1}^{p}(x)-\left(A_{n} x+B_{n}\right) r_{n}^{p}(x)+r_{n-1}^{p}(x)=\frac{R_{n}^{p}(x)}{N^{p+\frac{3}{2}}},
$$

where the nonhomogeneous term is given by

$$
\begin{aligned}
R_{n}^{p}(x) / N^{p+\frac{3}{2}}=\left(\frac{4 \zeta}{\left(\alpha_{0}^{\prime} x+\beta_{0}^{\prime}\right)^{2}-4}\right)^{\frac{1}{4}} & {\left[N^{\frac{1}{2}} I_{\nu}\left(N \zeta^{\frac{1}{2}}\right) F_{1, n}(x)\right.} \\
& \left.+N^{\frac{1}{2}} \zeta^{\frac{1}{2}} I_{\nu-1}\left(N \zeta^{\frac{1}{2}}\right) F_{2, n}(x)\right]
\end{aligned}
$$

with

$$
\begin{aligned}
F_{1, n}(x)= & G\left(\zeta, \frac{1}{N}\right) A_{p}\left(\zeta, \frac{1}{N+1}\right)+G\left(\zeta,-\frac{1}{N}\right) A_{p}\left(\zeta, \frac{1}{N-1}\right) \\
& -\Psi\left(x, \frac{1}{N}\right) A_{p}\left(\zeta, \frac{1}{N}\right)+\zeta L\left(\zeta, \frac{1}{N}\right) B_{p}\left(\zeta, \frac{1}{N+1}\right) \\
& -\zeta L\left(\zeta,-\frac{1}{N}\right) B_{p}\left(\zeta, \frac{1}{N-1}\right)
\end{aligned}
$$

and

$$
\begin{aligned}
F_{2, n}(x)= & H\left(\zeta, \frac{1}{N}\right) A_{p}\left(\zeta, \frac{1}{N+1}\right)-H\left(\zeta,-\frac{1}{N}\right) A_{p}\left(\zeta, \frac{1}{N-1}\right) \\
& -\Psi\left(x, \frac{1}{N}\right) B_{p}\left(\zeta, \frac{1}{N}\right)+K\left(\zeta, \frac{1}{N}\right) B_{p}\left(\zeta, \frac{1}{N+1}\right) \\
& +K\left(\zeta, \frac{1}{N}\right) B_{p}\left(\zeta, \frac{1}{N-1}\right) .
\end{aligned}
$$

Recall that the series

$$
A\left(\zeta, \frac{1}{N}\right)=\sum_{s=0}^{\infty} \frac{A_{s}(\zeta)}{N^{s}} \quad \text { and } \quad B\left(\zeta, \frac{1}{N}\right)=\sum_{s=0}^{\infty} \frac{B_{s}(\zeta)}{N^{s}}
$$

in (4.2) are formal solutions of (4.5) and (4.6). Since $A_{p}\left(\zeta, \frac{1}{N}\right)$ and $B_{p}\left(\zeta, \frac{1}{N}\right)$ can be written as

$$
A_{p}\left(\zeta, \frac{1}{N}\right)=\sum_{s=0}^{\infty} \frac{A_{s}^{*}(\zeta)}{N^{s}} \quad \text { and } \quad B_{p}\left(\zeta, \frac{1}{N}\right)=\sum_{s=0}^{\infty} \frac{B_{s}^{*}(\zeta)}{N^{s}}
$$

with $A_{s}^{*}(\zeta)=A_{s}(\zeta), B_{s}^{*}(\zeta)=B_{s}(\zeta)$ for $s \leq p$ and $A_{s}^{*}(\zeta)=B_{s}^{*}(\zeta)=0$ for $s \geq p+1$, terms with powers of $1 / N$ less than or equal to $p+1$ in the expansions of $F_{1, n}(x)$ and $F_{2, n}(x)$ all vanish. (Note: The recurrence relations (4.11) and (4.12) were obtained when we equated coefficients of $1 / N^{p}$ in (4.5) and (4.6) to zero.) Hence, using Lemma 2, it can be proved that there is a constant $C_{p}$ such that

$$
\left|F_{1, n}(x)\right| \leq C_{p}(1+|x|) / N^{p+2}
$$

and

$$
\left(1+\left|\zeta^{\frac{1}{2}}\right|\right)\left|F_{2, n}(x)\right| \leq C_{p}(1+|x|) / N^{p+2}
$$


for all $x \geq x_{-}+\delta$. From (5.7), it follows that

$$
\left|R_{n}^{p}(x)\right| \leq \widetilde{C}_{p}\left(\frac{4 \zeta}{\left(\alpha_{0}^{\prime} x+\beta_{0}^{\prime}\right)^{2}-4}\right)^{\frac{1}{4}}(1+|x|)\left[\left|I_{\nu}\left(N \zeta^{\frac{1}{2}}\right)\right|+\frac{|\zeta|^{\frac{1}{2}}}{1+|\zeta|^{\frac{1}{2}}}\left|I_{\nu-1}\left(N \zeta^{\frac{1}{2}}\right)\right|\right]
$$

for all $x \geq x_{-}+\delta$ and for some positive constant $\widetilde{C}_{p}$. Similarly, we have

$$
s_{n+1}^{p}(x)-\left(A_{n} x+B_{n}\right) s_{n}^{p}(x)+s_{n-1}^{p}(x)=\frac{S_{n}^{p}(x)}{N^{p+\frac{3}{2}}},
$$

where

$$
\left|S_{n}^{p}(x)\right| \leq \widetilde{C}_{p}\left(\frac{4 \zeta}{\left(\alpha_{0}^{\prime} x+\beta_{0}^{\prime}\right)^{2}-4}\right)^{\frac{1}{4}}(1+|x|)\left[\left|K_{\nu}\left(N \zeta^{\frac{1}{2}}\right)\right|+\frac{|\zeta|^{\frac{1}{2}}}{1+|\zeta|^{\frac{1}{2}}}\left|K_{\nu-1}\left(N \zeta^{\frac{1}{2}}\right)\right|\right] .
$$

We now establish the existence of two solutions $P_{n}(x)$ and $Q_{n}(x)$ of (1.3) satisfying

$$
P_{n}(x) \sim r_{n}^{0}(x) \text { and } Q_{n}(x) \sim s_{n}^{0}(x)
$$

as $n \rightarrow \infty$ for any fixed $x>x_{-}$. It is easily verified that

$$
\begin{array}{r}
r_{n}^{0}(x) \sim \frac{1}{\sqrt{2 \pi}}\left(\frac{4}{\left(\alpha_{0}^{\prime} x+\beta_{0}^{\prime}\right)^{2}-4}\right)^{\frac{1}{4}}\left[e^{-N \zeta^{\frac{1}{2}}+\left(\nu+\frac{1}{2}\right) \pi i}\left(A_{0}-\zeta^{\frac{1}{2}} B_{0}\right)\right. \\
\left.+e^{N \zeta^{\frac{1}{2}}}\left(A_{0}+\zeta^{\frac{1}{2}} B_{0}\right)\right], \quad x_{-}+\delta \leq x<x_{+},
\end{array}
$$

and

$$
s_{n}^{0}(x) \sim \sqrt{\frac{\pi}{2}}\left(\frac{4}{\left(\alpha_{0}^{\prime} x+\beta_{0}^{\prime}\right)^{2}-4}\right)^{\frac{1}{4}} e^{-N \zeta^{\frac{1}{2}}}\left(A_{0}-\zeta^{\frac{1}{2}} B_{0}\right), \quad x \geq x_{-}+\delta,
$$

as $n \rightarrow \infty$. If $P_{n}(x)$ and $Q_{n}(x)$ are two linearly independent solutions of (1.3) satisfying (5.15), then it follows from (5.16), (5.17) and (5.18) that

$$
P_{n+1}(x) Q_{n}(x)-P_{n}(x) Q_{n+1}(x)=P_{n+2}(x) Q_{n+1}(x)-P_{n+1}(x) Q_{n+2}(x)
$$

and

$$
\begin{aligned}
P_{n+1}(x) Q_{n}(x)-P_{n}(x) Q_{n+1}(x) & =\lim _{m \rightarrow \infty}\left[r_{m+1}^{0}(x) s_{m}^{0}(x)-r_{m}^{0}(x) s_{m+1}^{0}(x)\right] \\
& =A_{0}^{2}-\zeta B_{0}^{2} \neq 0 .
\end{aligned}
$$

Without loss of generality, we may assume $A_{0}^{2}-\zeta B_{0}^{2}=1$. From this, it follows that there exists a smooth function $\Gamma(\zeta)$ such that $\left(1+|\zeta|^{1 / 2}\right) \Gamma(\zeta)$ is bounded for $x \geq x_{-}+\delta$ and

$$
A_{0}=\cosh (\sqrt{\zeta} \Gamma(\zeta)), \quad B_{0}=\frac{\sinh (\sqrt{\zeta} \Gamma(\zeta))}{\sqrt{\zeta}}
$$

see the assumption in Lemma 2. With these choices, we have

$$
P_{n+1}(x) Q_{n}(x)-P_{n}(x) Q_{n+1}(x)=1 .
$$


This, in particular, shows that $P_{n}(x)$ and $Q_{n}(x)$ are two linearly independent solutions.

Now define

$$
\varepsilon_{n}^{p}(x):=P_{n}(x)-r_{n}^{p}(x) \quad \text { and } \quad \delta_{n}^{p}(x):=Q_{n}(x)-s_{n}^{p}(x) .
$$

We first show that the existence of $Q_{n}(x)$ to (1.3) satisfying (5.15) is equivalent to the existence of $\delta_{n}^{p}(x)$ to the summation formula

$$
\begin{aligned}
\delta_{n}^{p}(x)= & \sum_{j=n+1}^{\infty} \frac{\left[r_{n}^{p}(x) s_{j}^{p}(x)-s_{n}^{p}(x) r_{j}^{p}(x)\right] S_{j}^{p}(x)}{\left[r_{n+1}^{p}(x) s_{n}^{p}(x)-r_{n}^{p}(x) s_{n+1}^{p}(x)\right]\left(j+\tau_{0}\right)^{p+\frac{3}{2}}} \\
& +\sum_{j=n+1}^{\infty} \frac{\left[r_{n}^{p}(x) S_{j}^{p}(x)-s_{n}^{p}(x) R_{j}^{p}(x)\right] \delta_{j}^{p}(x)}{\left[r_{n+1}^{p}(x) s_{n}^{p}(x)-r_{n}^{p}(x) s_{n+1}^{p}(x)\right]\left(j+\tau_{0}\right)^{p+\frac{3}{2}}} .
\end{aligned}
$$

From (1.3) and (5.13), we obtain

$$
\delta_{n+1}^{p}(x)-\left(A_{n} x+B_{n}\right) \delta_{n}^{p}(x)+\delta_{n-1}^{p}(x)=-\frac{S_{n}^{p}(x)}{N^{p+\frac{3}{2}}} .
$$

Coupling (5.6) and (5.23) gives

$$
\begin{aligned}
r_{n}^{p}(x) \delta_{n+1}^{p}(x)-r_{n+1}^{p}(x) \delta_{n}^{p}(x)= & r_{n+1}^{p}(x) \delta_{n+2}^{p}(x)-r_{n+2}^{p}(x) \delta_{n+1}^{p}(x) \\
& +\frac{S_{n+1}^{p}(x) r_{n+1}^{p}(x)+R_{n+1}^{p}(x) \delta_{n+1}^{p}(x)}{(N+1)^{p+\frac{3}{2}}} .
\end{aligned}
$$

In exactly the same manner, we also have

$$
\begin{aligned}
s_{n}^{p}(x) \delta_{n+1}^{p}(x)-s_{n+1}^{p}(x) \delta_{n}^{p}(x)= & s_{n+1}^{p}(x) \delta_{n+2}^{p}(x)-s_{n+2}^{p}(x) \delta_{n+1}^{p}(x) \\
& +\frac{S_{n+1}^{p}(x) s_{n+1}^{p}(x)+S_{n+1}^{p}(x) \delta_{n+1}^{p}(x)}{(N+1)^{p+\frac{3}{2}}}
\end{aligned}
$$

and

$$
\begin{aligned}
r_{n+1}^{p}(x) s_{n}^{p}(x)-s_{n+1}^{p}(x) r_{n}^{p}(x)= & r_{n+2}^{p}(x) s_{n+1}^{p}(x)-s_{n+2}^{p}(x) r_{n+1}^{p}(x) \\
& +\frac{S_{n+1}^{p}(x) r_{n+1}^{p}(x)-R_{n+1}^{p}(x) s_{n+1}^{p}(x)}{(N+1)^{p+\frac{3}{2}}} .
\end{aligned}
$$

Repeated application of the last three equations yields, respectively,

$$
\begin{aligned}
r_{n}^{p}(x) \delta_{n+1}^{p}(x)-r_{n+1}^{p}(x) \delta_{n}^{p}(x)= & r_{m+1}^{p}(x) \delta_{m+2}^{p}(x)-r_{m+2}^{p}(x) \delta_{m+1}^{p}(x) \\
& +\sum_{j=n+1}^{m+1} \frac{R_{j}^{p}(x) \delta_{j}^{p}(x)+S_{j}^{p}(x) r_{j}^{p}(x)}{\left(j+\tau_{0}\right)^{p+\frac{3}{2}}}, \\
s_{n}^{p}(x) \delta_{n+1}^{p}(x)-s_{n+1}^{p}(x) \delta_{n}^{p}(x)= & s_{m+1}^{p}(x) \delta_{m+2}^{p}(x)-s_{m+2}^{p}(x) \delta_{m+1}^{p}(x) \\
& +\sum_{j=n+1}^{m+1} \frac{S_{j}^{p}(x) s_{j}^{p}(x)+S_{j}^{p}(x) \delta_{j}^{p}(x)}{\left(j+\tau_{0}\right)^{p+\frac{3}{2}}}
\end{aligned}
$$

and

$$
\begin{aligned}
r_{n+1}^{p}(x) s_{n}^{p}(x)-s_{n+1}^{p}(x) r_{n}^{p}(x)= & r_{m+2}^{p}(x) s_{m+1}^{p}(x)-s_{m+2}^{p}(x) r_{m+1}^{p}(x) \\
& +\sum_{j=n+1}^{m+1} \frac{S_{j}^{p}(x) r_{j}^{p}(x)-R_{j}^{p}(x) s_{j}^{p}(x)}{\left(j+\tau_{0}\right)^{p+\frac{3}{2}}} .
\end{aligned}
$$


If $Q_{n}(x)$ satisfies (5.15), then $\delta_{n}^{0}(x)=o\left(s_{n}^{0}(x)\right)$ as $n \rightarrow \infty$. Since $\delta_{n}^{p}(x)=\delta_{n}^{0}(x)+$ $s_{n}^{0}(x)-s_{n}^{p}(x)$ by $(5.21)$, and since $A_{s}(\zeta)$ and $\left(1+|\zeta|^{\frac{1}{2}}\right) B_{s}(\zeta)$ are bounded for $s=0, \ldots, p$ by Lemma 2 , we also have $\delta_{n}^{p}(x)=o\left(s_{n}^{0}(x)\right)$ as $n \rightarrow \infty$. In view of (5.16), (5.17) and (5.18), we have

$$
\begin{aligned}
r_{m+1}^{p}(x) \delta_{m+2}^{p}(x)-r_{m+2}^{p}(x) \delta_{m+1}^{p}(x) & \rightarrow 0, \\
s_{m+1}^{p}(x) \delta_{m+2}^{p}(x)-s_{m+2}^{p}(x) \delta_{m+1}^{p}(x) & \rightarrow 0
\end{aligned}
$$

and

$$
r_{m+2}^{p}(x) s_{m+1}^{p}(x)-s_{m+2}^{p}(x) r_{m+1}^{p}(x) \rightarrow 1
$$

as $m \rightarrow \infty$. By letting $m \rightarrow \infty$ in (5.27)-(5.29), we obtain

$$
\begin{aligned}
r_{n}^{p}(x) \delta_{n+1}^{p}(x)-r_{n+1}^{p}(x) \delta_{n}^{p}(x) & =\sum_{j=n+1}^{\infty} \frac{R_{j}^{p}(x) \delta_{j}^{p}(x)+S_{j}^{p}(x) r_{j}^{p}(x)}{\left(j+\tau_{0}\right)^{p+\frac{3}{2}}}, \\
s_{n}^{p}(x) \delta_{n+1}^{p}(x)-s_{n+1}^{p}(x) \delta_{n}^{p}(x) & =\sum_{j=n+1}^{\infty} \frac{S_{j}^{p}(x) s_{j}^{p}(x)+S_{j}^{p}(x) \delta_{j}^{p}(x)}{\left(j+\tau_{0}\right)^{p+\frac{3}{2}}}
\end{aligned}
$$

and

$$
r_{n+1}^{p}(x) s_{n}^{p}(x)-s_{n+1}^{p}(x) r_{n}^{p}(x)=1+\sum_{j=n+1}^{\infty} \frac{S_{j}^{p}(x) r_{j}^{p}(x)-R_{j}^{p}(x) s_{j}^{p}(x)}{\left(j+\tau_{0}\right)^{p+\frac{3}{2}}} .
$$

Upon solving (5.33) and (5.34), we obtain (5.22). The existence of a solution $\left\{\delta_{n}^{p}(x)\right\}_{n=1}^{\infty}$ to (5.22) is proved by using the successive approximation method. Starting with $\delta_{n, 0}^{p}(x)=0$, we define $\delta_{n, k}^{p}(x)$ by

$$
\begin{aligned}
\delta_{n, k}^{p}(x)= & \sum_{j=n+1}^{\infty} \frac{\left[r_{n}^{p}(x) s_{j}^{p}(x)-s_{n}^{p}(x) r_{j}^{p}(x)\right] S_{j}^{p}(x)}{\left[r_{n+1}^{p}(x) s_{n}^{p}(x)-r_{n}^{p}(x) s_{n+1}^{p}(x)\right]\left(j+\tau_{0}\right)^{p+\frac{3}{2}}} \\
& +\sum_{j=n+1}^{\infty} \frac{\left[r_{n}^{p}(x) S_{j}^{p}(x)-s_{n}^{p}(x) R_{j}^{p}(x)\right] \delta_{j, k-1}^{p}(x)}{\left[r_{n+1}^{p}(x) s_{n}^{p}(x)-r_{n}^{p}(x) s_{n+1}^{p}(x)\right]\left(j+\tau_{0}\right)^{p+\frac{3}{2}}}
\end{aligned}
$$

for $k \geq 1$. We shall show that for fixed $p$ and sufficiently large but also fixed $n$, the sequence $\left\{\delta_{n, k}^{p}(x)\right\}_{k \geq 0}$ is convergent as $k \rightarrow \infty$. Since $A_{s}(\zeta)$ and $\left(1+|\zeta|^{\frac{1}{2}}\right) B_{s}(\zeta)$ are bounded for $x \geq x_{-}+\delta$, it follows from (5.3)-(5.5) that

$$
\left|r_{n}^{p}(x)\right| \leq C N^{\frac{1}{2}}\left(\frac{4 \zeta}{\left(\alpha_{0}^{\prime} x+\beta_{0}^{\prime}\right)^{2}-4}\right)^{\frac{1}{4}}\left[\left|I_{\nu}\left(N \zeta^{\frac{1}{2}}\right)\right|+\frac{\left|\zeta^{\frac{1}{2}}\right|}{1+\left|\zeta^{\frac{1}{2}}\right|}\left|I_{\nu-1}\left(N \zeta^{\frac{1}{2}}\right)\right|\right]
$$

and

$$
\left|s_{n}^{p}(x)\right| \leq C N^{\frac{1}{2}}\left(\frac{4 \zeta}{\left(\alpha_{0}^{\prime} x+\beta_{0}^{\prime}\right)^{2}-4}\right)^{\frac{1}{4}}\left[\left|K_{\nu}\left(N \zeta^{\frac{1}{2}}\right)\right|+\frac{\left|\zeta^{\frac{1}{2}}\right|}{1+\left|\zeta^{\frac{1}{2}}\right|}\left|K_{\nu-1}\left(N \zeta^{\frac{1}{2}}\right)\right|\right]
$$

for some positive constant $C$. Furthermore, by virtue of the behaviors of $I_{\nu}$ and $K_{\nu}$, we have from (5.12) and (5.14)

$$
\left|R_{n}^{p}(x) s_{n}^{p}(x)\right| \leq M^{\prime} N^{\frac{1}{2}}\left(\frac{1+\left|\zeta^{\frac{1}{2}}\right|}{1+N\left|\zeta^{\frac{1}{2}}\right|}\right) \leq M^{\prime} N^{\frac{1}{2}}
$$

and

$$
\left|S_{n}^{p}(x) r_{n}^{p}(x)\right| \leq M^{\prime} N^{\frac{1}{2}}\left(\frac{1+\left|\zeta^{\frac{1}{2}}\right|}{1+N\left|\zeta^{\frac{1}{2}}\right|}\right) \leq M^{\prime} N^{\frac{1}{2}}
$$


where $M^{\prime}$ is a positive constant. Thus, from (5.35) we obtain

$$
\left|r_{n+1}^{p}(x) s_{n}^{p}(x)-s_{n+1}^{p}(x) r_{n}^{p}(x)-1\right| \leq \frac{2 M^{\prime}}{p} \cdot \frac{1}{N^{p}},
$$

which in turn gives

$$
r_{n+1}^{p}(x) s_{n}^{p}(x)-s_{n+1}^{p}(x) r_{n}^{p}(x)>\frac{1}{2}
$$

for large $n$, say $n>4 M^{\prime}-\tau_{0}$. A combination of (5.36), (5.37), (5.38) and (5.41) yields

$$
\begin{aligned}
& \left|\delta_{n, 1}^{p}(x)\right| \\
& \quad \leq 2 \sum_{j=n+1}^{\infty} \frac{\left[\left|r_{n}^{p}(x) s_{j}^{p}(x)\right|+\left|s_{n}^{p}(x) r_{j}^{p}(x)\right|\right]\left|S_{j}^{p}(x)\right|}{\left(j+\tau_{0}\right)^{p+\frac{3}{2}}} \\
& \quad \leq M^{\prime \prime}\left(\frac{4 \zeta}{\left(\alpha_{0}^{\prime} x+\beta_{0}^{\prime}\right)^{2}-4}\right)^{\frac{1}{4}}\left(\sum_{j=n+1}^{\infty} \frac{N^{\frac{1}{2}}}{\left(j+\tau_{0}\right)^{p+1}}\right)\left[\left|K_{\nu}\left(N \zeta^{\frac{1}{2}}\right)\right|+\left|K_{\nu-1}\left(N \zeta^{\frac{1}{2}}\right)\right|\right]
\end{aligned}
$$

for some constant $M^{\prime \prime}>0$, where we have also used (5.40) and the monotonicity properties of $I_{\nu}$ and $K_{\nu}$. Hence

$$
\left|\delta_{n, 1}^{p}(x)\right| \leq \frac{M^{\prime \prime}}{p} \cdot \frac{1}{N^{p-\frac{1}{2}}}\left(\frac{4 \zeta}{\left(\alpha_{0}^{\prime} x+\beta_{0}^{\prime}\right)^{2}-4}\right)^{\frac{1}{4}}\left[\left|K_{\nu}\left(N \zeta^{\frac{1}{2}}\right)\right|+\left|K_{\nu-1}\left(N \zeta^{\frac{1}{2}}\right)\right|\right]
$$

Similarly, we can prove by induction that

$$
\begin{aligned}
\left|\delta_{n, k}^{p}(x)-\delta_{n, k-1}^{p}(x)\right| \leq & \left(\frac{M^{\prime \prime}}{p} \cdot \frac{1}{N^{p}}\right)^{k}\left(\frac{4 \zeta}{\left(\alpha_{0}^{\prime} x+\beta_{0}^{\prime}\right)^{2}-4}\right)^{\frac{1}{4}} N^{\frac{1}{2}} \\
& {\left[\left|K_{\nu}\left(N \zeta^{\frac{1}{2}}\right)\right|+\left|K_{\nu-1}\left(N \zeta^{\frac{1}{2}}\right)\right|\right] }
\end{aligned}
$$

from which it also follows that

$$
\delta_{n, k}^{p}(x)=\sum_{m=1}^{k}\left[\delta_{n, m}^{p}(x)-\delta_{n, m-1}^{p}(x)\right]
$$

converges, as $k \rightarrow \infty$, for all $n \geq 2 M^{\prime \prime}-\tau_{0}$. Clearly, the limit function $\delta_{n}^{p}(x)$ satisfies (5.22). Thus, $Q_{n}(x)=s_{n}^{p}(x)+\delta_{n}^{p}(x)$ is a solution of (1.3) satisfying (5.15). Furthermore, we have from (5.42), (5.43) and (5.44) that

$$
\left|\delta_{n}^{p}(x)\right| \leq M_{p}^{\prime}\left(\frac{4 \zeta}{\left(\alpha_{0}^{\prime} x+\beta_{0}^{\prime}\right)^{2}-4}\right)^{\frac{1}{4}}\left[\left|K_{\nu}\left(N \zeta^{\frac{1}{2}}\right)\right|+\left|K_{\nu-1}\left(N \zeta^{\frac{1}{2}}\right)\right|\right] / N^{p-\frac{1}{2}} .
$$

Note that $A_{s}(\zeta)$ and $\left(1+\left|\zeta^{\frac{1}{2}}\right|\right) B_{s}(\zeta)$ are bounded for $x \geq x_{-}+\delta$. By taking an extra term in the expansion (5.5), we have

$$
\begin{aligned}
\left|\delta_{n}^{p}(x)\right| \leq\left|\delta_{n}^{p+1}(x)\right|+M_{p}^{\prime}\left(\frac{4 \zeta}{\left(\alpha_{0}^{\prime} x+\beta_{0}^{\prime}\right)^{2}-4}\right)^{\frac{1}{4}} & {\left[\left|K_{\nu}\left(N \zeta^{\frac{1}{2}}\right)\right|\left|\frac{A_{p+1}(\zeta)}{N^{p+\frac{1}{2}}}\right|\right.} \\
& \left.+\left|\zeta^{\frac{1}{2}} K_{\nu-1}\left(N \zeta^{\frac{1}{2}}\right)\right|\left|\frac{B_{p+1}(\zeta)}{N^{p+\frac{1}{2}}}\right|\right] \\
\leq & M_{p}\left(\frac{4 \zeta}{\left(\alpha_{0}^{\prime} x+\beta_{0}^{\prime}\right)^{2}-4}\right)^{\frac{1}{4}}\left[\left|K_{\nu}\left(N \zeta^{\frac{1}{2}}\right)\right|+\left|K_{\nu-1}\left(N \zeta^{\frac{1}{2}}\right)\right|\right] / N^{p+\frac{1}{2}}
\end{aligned}
$$


for some positive constants $M_{p}^{\prime}$ and $M_{p}$, and (2.20) follows; see also (5.21).

The proof of (2.19) is very similar. As in (5.34), we have

$$
Q_{n}(x) \varepsilon_{n+1}^{p}(x)-Q_{n+1}(x) \varepsilon_{n}^{p}(x)=\sum_{j=n+1}^{\infty} \frac{R_{j}^{p}(x) Q_{j}(x)}{\left(j+\tau_{0}\right)^{p+\frac{3}{2}}} .
$$

Since $P_{n}(x) \varepsilon_{n+1}^{p}(x)-P_{n+1}(x) \varepsilon_{n}^{p}(x)=r_{n}^{p}(x) \varepsilon_{n+1}^{p}(x)-r_{n+1}^{p}(x) \varepsilon_{n}^{p}(x)$, it follows from (5.6) that

$$
\begin{aligned}
P_{n}(x) \varepsilon_{n+1}^{p}(x)-P_{n+1}(x) \varepsilon_{n}^{p}(x) & =r_{m}^{p}(x) \varepsilon_{m+1}^{p}(x)-r_{m+1}^{p}(x) \varepsilon_{m}^{p}(x) \\
& -\sum_{j=m+1}^{n} \frac{R_{j}^{p}(x) r_{j}^{p}(x)+R_{j}^{p}(x) \varepsilon_{j}^{p}(x)}{\left(j+\tau_{0}\right)^{p+\frac{3}{2}}},
\end{aligned}
$$

where $n>m$; cf. (5.28). We may choose $m=[n / 2]$. Upon solving the last two equations, we get from (5.20)

$$
\begin{aligned}
\varepsilon_{n}^{p}(x)= & {\left[r_{m+1}^{p}(x) \varepsilon_{m}^{p}(x)-r_{m}^{p}(x) \varepsilon_{m+1}^{p}(x)\right] Q_{n}(x) } \\
& +\sum_{j=n+1}^{\infty} \frac{\left[r_{n}^{p}(x)+\varepsilon_{n}^{p}(x)\right] R_{j}^{p}(x) Q_{j}(x)}{\left(j+\tau_{0}\right)^{p+\frac{3}{2}}} \\
& +\sum_{j=m+1}^{\infty} \frac{\left[R_{j}^{p}(x) r_{j}^{p}(x)+R_{j}^{p}(x) \varepsilon_{j}^{p}(x)\right] Q_{n}(x)}{\left(j+\tau_{0}\right)^{p+\frac{3}{2}}} .
\end{aligned}
$$

The estimate

$$
\left|\varepsilon_{n}^{p}(x)\right| \leq M_{p}\left(\frac{4 \zeta}{\left(\alpha_{0}^{\prime} x+\beta_{0}^{\prime}\right)^{2}-4}\right)^{\frac{1}{4}}\left[\left|I_{\nu}\left(N \zeta^{\frac{1}{2}}\right)\right|+\left|I_{\nu-1}\left(N \zeta^{\frac{1}{2}}\right)\right|\right] / N^{p+\frac{1}{2}}
$$

is again proved by using the successive approximation method.

\section{An example}

As an illustration, we consider monic polynomials $\pi_{n}(x)$ that are orthogonal on $[-1,1]$ with respect to a modified Jacobi weight $w(x)=(1-x)^{\alpha}(1+x)^{\beta} h(x)$, where $\alpha, \beta>-1$ and $h$ is real analytic and strictly positive on $[-1,1]$. These polynomials satisfy a three-term recurrence relation

$$
\pi_{n+1}(x)=\left(x-b_{n}\right) \pi_{n}(x)-a_{n}^{2} \pi_{n-1}(x),
$$

where the coefficients $a_{n}$ and $b_{n}$ have asymptotic expansions of the form

$$
\begin{gathered}
a_{n} \sim \frac{1}{2}+\sum_{k=2}^{\infty} \frac{C_{k}}{n^{k}}, \\
b_{n} \sim \sum_{k=2}^{\infty} \frac{D_{k}}{n^{k}} .
\end{gathered}
$$


The first few coefficients $C_{k}$ and $D_{k}$ are given by

$$
\begin{aligned}
& C_{2}=-\frac{4 \alpha^{2}-1}{32}-\frac{4 \beta^{2}-1}{32}, \\
& C_{3}=\frac{4 \alpha^{2}-1}{32}\left(\alpha+\beta+c_{0}\right)+\frac{4 \beta^{2}-1}{32}\left(\alpha+\beta+d_{0}\right), \\
& D_{2}=-\frac{\beta^{2}-\alpha^{2}}{4}, \\
& D_{3}=-\frac{\beta^{2}-\alpha^{2}}{4}(1+\alpha+\beta)+c_{0} \frac{4 \alpha^{2}-1}{16}-d_{0} \frac{4 \beta^{2}-1}{16},
\end{aligned}
$$

where

$$
\begin{aligned}
& c_{0}=\frac{1}{2 \pi i} \int_{\gamma} \frac{\log h(t)}{\sqrt{t^{2}-1}} \frac{d t}{t-1}, \\
& d_{0}=\frac{1}{2 \pi i} \int_{\gamma} \frac{\log h(t)}{\sqrt{t^{2}-1}} \frac{d t}{t+1},
\end{aligned}
$$

and $\gamma$ is a closed contour encircling the interval $[-1,1]$ once in the positive direction; see Kuijlaars et al. [12], where an asymptotic expansion for the polynomials $\pi_{n}(x)$ has also been given, which holds uniformly in compact subsets of $\mathbb{C} \backslash[-1,1]$. Moreover, in another paper [13, Kuijlaars and Vanlessen have presented a uniform asymptotic expansion for $\pi_{n}(x)$ in the interval $(-1+\delta, 1-\delta)$, which agrees with the one given by Szegö [17, p. 298, Theorem 12.1.6 and footnote 59]:

$$
\pi_{n}(x)=\frac{\sqrt{2} D_{\infty}}{2^{n} w^{\frac{1}{2}}(x)\left(1-x^{2}\right)^{\frac{1}{4}}}[\cos (n \arccos x+\gamma(x))+o(1)]
$$

where

$$
D_{\infty}=\exp \left(\frac{1}{2 \pi} \int_{-1}^{1} \frac{\log w(t)}{\sqrt{1-t^{2}}} d t\right)
$$

and

$$
\gamma(x)=\frac{\left(1-x^{2}\right)^{\frac{1}{2}}}{2 \pi} P V \int_{-1}^{1} \frac{\log \left[\sqrt{1-t^{2}} w(t)\right]}{\sqrt{1-t^{2}}} \frac{d t}{t-x} .
$$

Here the integral is taken in the Cauchy principal-value sense. Note that the weight function $w(x)$ satisfies the Szegö condition

$$
\int_{-1}^{1} \frac{\log w(x)}{\sqrt{1-x^{2}}} d x>-\infty
$$

and that we have

$$
\lim _{n \rightarrow \infty} \frac{2^{n} \pi_{n}(z)}{\varphi^{n}(z)}=\frac{D_{\infty}}{D(z)} \frac{\varphi^{\frac{1}{2}}(z)}{\sqrt{2}\left(z^{2}-1\right)^{\frac{1}{4}}},
$$

holding uniformly for $z$ in compact subsets of $\mathbb{C} \backslash[-1,1]$, where

$$
\varphi(z)=z+\sqrt{z^{2}-1}, \quad z \in \mathbb{C} \backslash[-1,1],
$$

and

$$
D(z)=\exp \left(-\frac{\left(z^{2}-1\right)^{\frac{1}{2}}}{2 \pi} \int_{-1}^{1} \frac{\log w(t)}{\sqrt{1-t^{2}}} \frac{d t}{t-z}\right) .
$$


In (6.14) and (6.15), we take that branch of $\left(z^{2}-1\right)^{1 / 2}$ which is analytic in $\mathbb{C} \backslash[-1,1]$ and behaves like $z$ as $z \rightarrow \infty$. The function $\varphi(z)$ in (6.14) is the familiar Joukowski or aerofoil map that maps the exterior of $[-1,1]$ conformally onto the exterior of the unit ball, and the function $D(z)$ is the so-called Szegö function associated with the weight $w(x)$; see [17, p. 277].

Returning to the three-term recurrence relation (6.1), we let

$$
K_{n}=2^{-n} \prod_{m=0}^{\infty}\left(2 a_{n+2 m+1}\right)^{-2} .
$$

It can be readily verified that $K_{n+1} / K_{n-1}=a_{n}^{2}$ and $P_{n}(x):=\pi_{n}(x) / K_{n}$ satisfies the recurrence relation (1.3)

$$
P_{n+1}(x)-\left(A_{n} x+B_{n}\right) P_{n}(x)+P_{n-1}=0
$$

with $A_{n}=K_{n} / K_{n+1}$ and $B_{n}=-b_{n} K_{n} / K_{n+1}$. From (6.2)-(6.7), it follows that the coefficients $A_{n}$ and $B_{n}$ satisfy

$$
A_{n} \sim 2+\sum_{s=2}^{\infty} \frac{\alpha_{s}}{n^{s}} \quad \text { and } \quad B_{n} \sim \sum_{s=2}^{\infty} \frac{\beta_{s}}{n^{s}},
$$

where

$$
\begin{aligned}
& \alpha_{2}=\frac{1}{4}\left(2 \alpha^{2}+2 \beta^{2}-1\right), \\
& \alpha_{3}=-\frac{4 \alpha^{2}-1}{8}\left(\alpha+\beta+1+c_{0}\right)-\frac{4 \beta^{2}-1}{8}\left(\alpha+\beta+1+d_{0}\right), \\
& \beta_{2}=\frac{\alpha^{2}-\beta^{2}}{2}
\end{aligned}
$$

and

$$
\beta_{3}=-\frac{4 \alpha^{2}-1}{8}\left(\alpha+\beta+1+c_{0}\right)+\frac{4 \beta^{2}-1}{8}\left(\alpha+\beta+1+d_{0}\right) .
$$

In terms of the notation in (2.3), (2.14) and (4.20), we have

$$
x_{+}=1, \quad x_{-}=-1, \quad \nu=\alpha
$$

and

$$
N=n+\tau_{0}=n+\frac{\alpha+\beta+1+c_{0}}{2} .
$$

By our main theorem, there are two linearly independent solutions

$$
P_{n}(x) \sim\left(\frac{\zeta}{x^{2}-1}\right)^{\frac{1}{4}}\left[N^{\frac{1}{2}} I_{\alpha}\left(N \zeta^{\frac{1}{2}}\right) \sum_{s=0}^{\infty} \frac{A_{s}(\zeta)}{N^{s}}+N^{\frac{1}{2}} \zeta^{\frac{1}{2}} I_{\alpha-1}\left(N \zeta^{\frac{1}{2}}\right) \sum_{s=0}^{\infty} \frac{B_{s}(\zeta)}{N^{s}}\right]
$$

and

$$
Q_{n}(x) \sim\left(\frac{\zeta}{x^{2}-1}\right)^{\frac{1}{4}}\left[N^{\frac{1}{2}} K_{\alpha}\left(N \zeta^{\frac{1}{2}}\right) \sum_{s=0}^{\infty} \frac{A_{s}(\zeta)}{N^{s}}-N^{\frac{1}{2}} \zeta^{\frac{1}{2}} K_{\alpha-1}\left(N \zeta^{\frac{1}{2}}\right) \sum_{s=0}^{\infty} \frac{B_{s}(\zeta)}{N^{s}}\right]
$$

for $x \geq-1+\delta$, where

$$
\zeta^{\frac{1}{2}}(x)=\log \varphi(x)=\log \left(x+\sqrt{x^{2}-1}\right)
$$


for $x \geq 1$ and

$$
\zeta^{\frac{1}{2}}(x)=\mathrm{e}^{\frac{\pi}{2} i} \arccos x
$$

for $-1<x<1$; see (4.8) and (6.14). Since $\pi_{n}(x) / K_{n}$ is also a solution of (1.3), there exist two functions $C_{1}(x)$ and $C_{2}(x)$, which are independent of $n$, such that

$$
\pi_{n}(x) / K_{n}=C_{1}(x) P_{n}(x)+C_{2}(x) Q_{n}(x) .
$$

To determine these two functions, we first choose the function $\Gamma(\zeta)$ in $(5.19)$ as

$$
\Gamma(\zeta)=\left(\frac{z^{2}-1}{\zeta}\right)^{\frac{1}{2}} \frac{1}{4 \pi i} \int_{\gamma} \frac{\log h(t)}{\sqrt{t^{2}-1}} \frac{d t}{t-z}-\frac{c_{0}}{2}, \quad z \in \mathbb{C} \backslash(-\infty,-1],
$$

where $\zeta$ is the analytic function of $z$ given in (6.33) and $\gamma$ is a closed contour encircling the interval $[-1,1]$ in the positive direction and also the given point $z$. Obviously, $\Gamma(\zeta)$ is analytic in $z \in \mathbb{C} \backslash(-\infty,-1]$. From (6.12) and (6.26), it is clear that

$$
\Gamma(\zeta(x)) \arccos x=\gamma(x)+\frac{\alpha \pi}{2}+\frac{\pi}{4}-\frac{1}{2}\left(\alpha+\beta+1+c_{0}\right) \arccos x
$$

for $-1<x<1$. From (5.17) and (5.18), we have

$$
P_{n}(x) \sim \sqrt{\frac{2}{\pi}}\left(1-x^{2}\right)^{-\frac{1}{4}} \mathrm{e}^{\alpha \pi i / 2} \cos \left[(N+\Gamma(\zeta)) \arccos x-\frac{\alpha \pi}{2}-\frac{\pi}{4}\right]
$$

and

$$
\begin{aligned}
Q_{n}(x) \sim \sqrt{\frac{\pi}{2}}\left(1-x^{2}\right)^{-\frac{1}{4}} \mathrm{e}^{-(\alpha+1) \pi i / 2}\{ & \cos \left[(N+\Gamma(\zeta)) \arccos x-\frac{\alpha \pi}{2}-\frac{\pi}{4}\right] \\
& \left.-i \sin \left[(N+\Gamma(\zeta)) \arccos x-\frac{\alpha \pi}{2}-\frac{\pi}{4}\right]\right\}
\end{aligned}
$$

as $n \rightarrow \infty$, for $-1<x<1$. Letting $n \rightarrow \infty$ in (6.25), it follows from (6.10) and (6.27) that

(6.30) $\pi_{n}(x) / K_{n} \sim \sqrt{2} D_{\infty} w^{-\frac{1}{2}}(x)\left(1-x^{2}\right)^{-\frac{1}{4}} \cos \left[(N+\Gamma(\zeta)) \arccos x-\frac{\alpha \pi}{2}-\frac{\pi}{4}\right]$.

A combination of (6.25), (6.28), (6.29) and (6.30) gives

$$
C_{1}(x)=\sqrt{\pi} D_{\infty} w^{-\frac{1}{2}}(x) \mathrm{e}^{-\frac{\alpha \pi}{2} i} \quad \text { and } \quad C_{2}(x)=0
$$

for $-1<x<1$. Thus, we obtain

$$
\pi_{n}(x) / K_{n}=\sqrt{\pi} D_{\infty}(1+x)^{-\frac{\beta}{2}} h^{-\frac{1}{2}}(x)\left[\mathrm{e}^{-\frac{\alpha \pi}{2} i}(1-x)^{-\frac{\alpha}{2}} P_{n}(x)\right]
$$

for $-1<x<1$. Note that $\mathrm{e}^{-\alpha \pi i / 2}(1-x)^{-\alpha / 2} I_{\alpha}\left(N(-\zeta)^{1 / 2} i\right)$ can be written as $(x-1)^{-\alpha / 2} I_{\alpha}\left(N \zeta^{1 / 2}\right)$, where

$$
\zeta^{\frac{1}{2}}(z):=\log \left(z+\sqrt{z^{2}-1}\right), \quad z \in \mathbb{C} \backslash(-\infty, 1],
$$

and also note that all functions $(z-1)^{-\alpha / 2} I_{\alpha}\left(N \zeta^{1 / 2}\right),(z-1)^{-\alpha / 2} P_{n}(z)$ and $(z+1)^{-\beta / 2} h^{-1 / 2}(z)$ are analytic in $z \in \mathbb{C} \backslash(-\infty,-1]$. By analytic continuation, we have from $(6.32)$

$$
\pi_{n}(z) / K_{n}=\sqrt{\pi} D_{\infty}(z+1)^{-\frac{\beta}{2}} h^{-\frac{1}{2}}(z)\left[(z-1)^{-\frac{\alpha}{2}} P_{n}(z)\right]
$$


for $z \in \mathbb{C} \backslash(-\infty,-1]$. In particular, when $z=x=\cos \theta, \theta \in(0, \pi-\delta)$, we obtain the uniform asymptotic expansion

$$
\begin{aligned}
& w^{\frac{1}{2}}(\cos \theta)\left(\frac{\sin \theta}{\theta}\right)^{\frac{1}{2}} \pi_{n}(\cos \theta) \\
& \sim K_{n} \sqrt{\pi} D_{\infty}\left[N^{\frac{1}{2}} J_{\alpha}(N \theta) \sum_{s=0}^{\infty} \frac{A_{s}\left(-\theta^{2}\right)}{N^{s}}-N^{\frac{1}{2}} \theta J_{\alpha-1}(N \theta) \sum_{s=0}^{\infty} \frac{B_{s}\left(-\theta^{2}\right)}{N^{s}}\right],
\end{aligned}
$$

where $J_{\alpha}$ is the Bessel function of first kind.

\section{REFERENCES}

[1] G. D. Birkhoff, Formal theory of irregular linear difference equations, Acta Math., 54 (1930), pp. 205-246.

[2] G. D. Birkhoff and W. J. Trjitzinsky, Analytic theory of singular difference equations, Acta Math., 60 (1932), pp. 1-89.

[3] P. Bleher and A. Its, Semiclassical asymptotics of orthogonal polynomials, Riemann-Hilbert problem, and universality in the matrix model, Ann. Math., 150 (1999), pp. 185-266. MR 2000k:42033

[4] P. Deift, T. Kriecherbauer, K. T-R McLaughlin, S. Venakides, and X. Zhou, Uniform asymptotics for polynomials orthogonal with respect to varying exponential weights and applications to universality questions in random matrix theory, Comm. Pure Appl. Math., 52 (1999), pp. 1335-1425. MR 2001g:42050

[5] P. Deift, T. Kriecherbauer, K. T-R McLaughlin, S. Venakides, and X. Zhou, Strong asymptotics of orthogonal polynomials with respect to exponential weights, Comm. Pure Appl. Math., 52 (1999), pp. 1491-1552. MR 2001f:42037

[6] P. Deift, T. Kriecherbauer, K. T-R McLaughlin, S. Venakides, and X. Zhou, A RiemannHilbert approach to asymptotic questions for orthogonal polynomials, J. Comput. Appl. Math., 133 (2001), pp. 47-63. MR 2002h:42044

[7] P. Deift and X. Zhou, A steepest descent method for oscillatory Riemann-Hilbert problems, Applications for the MKdV equation, Ann. Math., 137 (1993), pp. 295-368. MR 94d:35143

[8] R. B. Dingle and G. J. Morgan, WKB methods for difference equations I, Appl. Sci. Res., 18 (1967), pp. 221-237. MR 37:1104

[9] R. B. Dingle and G. J. Morgan, WKB methods for difference equations II, Appl. Sci. Res., 18 (1967), pp. 238-245. MR 37:1104

[10] T. Kriecherbauer and K. T-R McLaughlin, Strong asymptotics of polynomials orthogonal with respect to Freud weights, Internat. Math. Res. Notices (1999), pp. 299-333. MR 2000h:33016

[11] A. B. J. Kuijlaars and K. T-R McLaughlin, Riemann-Hilbert analysis for Laguerre polynomials with large negative parameter, Comput. Meth. Funct. Theory, 1 (2001), pp. 205-233. MR 2003k:30059

[12] A. B. J. Kuijlaars, K. T-R McLaughlin, W. Van Assche, and M. Vanlessen, The RiemannHilbert approach to strong asymptotics for orthogonal polynomials on $[-1,1]$, preprint math. CA/0111252.

[13] A. B. J. Kuijlaars and M. Vanlessen, Universality for eigenvalue correlations from the modified Jacobi unitary ensemble, Internat. Math. Res. Notices (2002), pp. 1575-1600. MR 2003g:30043

[14] X. Li and R. Wong, On the asymptotics of the Meixner-Pollaczek polynomials and their zeros, Constr. Approx., 17 (2001), pp. 59-90. MR 2001m:33012

[15] F. W. J. Olver, Asymptotics and Special Functions, Academic Press, New York, 1974. (Reprinted by A. K. Peters Ltd., Wellesley, 1997.) MR 97i:41001

[16] W.-Y. Qiu and R. Wong, Uniform asymptotic formula for orthogonal polynomials with exponential weight, SIAM J. Math. Anal., 31 (2000), pp. 992-1029. MR 2001f:42039

[17] G. Szegö, "Orthogonal Polynomials," Fourth edition, Colloquium Publications, Vol. 23, Amer. Math. Soc. Providence R. I., 1975. MR 51:8724 
[18] W. Van Assche, J. S. Geronimo, and A. B. J. Kuijlaars, Riemann-Hilbert problems for multiple orthogonal polynomials, pp. 23-50 in "NATO ASI Special Functions 2000" (J. Bustoz et. al. eds.), Kluwer Academic Publisher, Dordrecht, 2001.

[19] Z. Wang and R. Wong, Uniform asymptotic expansion of $J_{\nu}(\nu a)$ via a difference equation, Numer. Math., 91 (2002), pp. 147-193. MR 2003g:33008

[20] Z. Wang and R. Wong, Asymptotic expansions for second-order linear difference equations with a turning point, Numer. Math., 94 (2003), pp. 147-194.

[21] R. Wong and H. Li, Asymptotic expansions for second-order linear difference equations, J. Comput. Appl. Math., 41 (1992), pp. 65-94. MR 94i:39003

[22] R. Wong and H. Li, Asymptotic expansions for second-order linear difference equations II, Stud. Appl. Math., 87 (1992), pp. 289-324. MR 94i:39004

Department of Mathematics, City University of Hong Kong, Tat Chee Avenue, Kowloon, Hong Kong; Wuhan Institute of Physics and Mathematics, The Chinese Academy of Sciences, P. O. Box 71010, Wuhan 430071, Peoples Republic of China

E-mail address: mcwang@cityu.edu.hk

Department of Mathematics, City University of Hong Kong, Tat Chee Avenue, KOWLOON, Hong Kong

E-mail address: mawong@cityu.edu.hk 